\title{
A Space-Time Finite Element Approximation of a Two-Step Chemical Kinetics Model
}

\author{
Abdellatif Agouzal ${ }^{1}$, Karam Allali $^{2, *}$ \\ ${ }^{1}$ Department of Mathematics, Institut Camille Jordan, University Lyon I, 43 Bd 11 November, 69100 Villeurbanne, France \\ ${ }^{2}$ Department of Mathematics, Faculty of Sciences and Technologies, University Hassan II, Po.Box 146, Mohammedia, Morocco \\ *Corresponding Author: allali@fstm.ac.ma
}

Copyright (C2013 Horizon Research Publishing All rights reserved.

\begin{abstract}
In this paper we suggest a fully discretized problem of a model describing two-step chemical kinetics. The model considered is a system of equations coupling Navier-Stokes equations with three non-linear reaction-diffusion equations. A space-time finite elements approximations are presented. The stability of the fully discretized problem is studied. Optimal error estimates are given.
\end{abstract}

Keywords A priori error estimates, Boussinesq approximation, Chemical kinetics, Mixed finite elements

\section{Introduction}

Chemical kinetics can be defined as the study of chemical or biological systems that its compositions change with time. For example, in biological systems, the cellular behaviour involves a very large number of chemical reactions, usually, catalyzed by proteins. Those reactions, for simplicity, can be described as a simple chemical reaction between two or more states of the system. Several works have been devoted to chemical kinetics, see for instance $[5,6,11,17]$.

The numerical analysis of one-step chemical kinetics is studied in [1]. The authors prove the existence, uniqueness of the solutions and also give some a priori error estimates. In this paper, we are interested in a two-step chemical kinetics. In the first step, the specie $A$ gives a product $B$ which undergoes a second reaction and gives the specie $C$ as result of the second step. For example, in biology reactions, $A$ could be the unfolded state of a protein, $B$ the compact denatured state or "molten globule" state and $C$ the final native state of the protein [4, 9].

It is worth noticing that when two time curves $A$ and $C$ or $B$ are known, the solution is easy, but in many cases the simultaneous determination of the substrate, intermediate and the final product is difficult or impossible. So, it is very significant to develop a numerical research method for this kind of complex reaction.

We consider both steps be exothermic first-order reactions with the corresponding reaction rates, given by the Arrhenius law [18]: $k_{i}=k_{i_{0}} \exp \left(E_{i} / R T\right)$, here $E_{i}$ is the activation energy, $k_{i_{0}}$ the pre-exponential factor, $R$ the universal gas constant, $T$ the temperature and the index $i$ denotes the step number $(i=1,2)$. It was established that when the activation energy of the first reaction is much greater than the activation energy of the second reaction (the second reaction is faster that the first) the second specie $B$ will be immediately consumed, as quickly as it is formed. In the other case, when the second reaction is slower than the first one, all the two reactants and the two products will appear (it is the case of Nitrogen dioxide which can give the final product Nitrogen via the intermediate specie Nitrogen monoxide) [16, 19]. Same phenomenon is observed in some biochemistry problems when using Michaelis-Menten kinetics [15], in the model, the unknowns are the concentrations of enzymes.

Perhaps the simplest model of such process can be described by the following system of differential equations $[13]$ :

$$
\begin{array}{r}
\frac{d[A]}{d t}=-k_{1}[A], \\
\frac{d[B]}{d t}=k_{1}[A]-k_{2}[B] .
\end{array}
$$

Here $[A]$ and $[B]$ are respectively the concentrations of species $A$ and $B$. It is well known that the concentration of the final product is given by: $1-[A]-[B]$. In this paper, we will take into account the influence of the 
hydrodynamic and energy on those kind of reactions. To this end, we will consider a more generalized model. The semi-descritized problem of such model has been studied in [2]. The authors prove the existence of the solution and give the a priori error estimates on the different unknowns of the model. This work is devoted to the fully discretized problem.

The paper is organized as follows. In Section 2, we give the model describing the two-step chemical kinetics, in Section 3 and 4, we give our variational problem and study its stability. In the Section 5, we give the a priori error estimates on the different unknowns of the problem.

\section{Model presentation}

For our two-step chemical kinetics, we will consider the incompressibility assumption on all the reactants and the priducts; therefore, we will consider a coupling between the hydrodynamic and the reaction-diffusion equations, under the classical Boussinesq approximation [14].

The model for such process, is given by:

$$
\left\{\begin{array}{l}
\partial_{t} T-\lambda \Delta T+u . \nabla T-C_{1} g_{1}(T)-C_{2} g_{2}(T)=0 \\
\partial_{t} C_{1}-d_{1} \Delta C_{1}+u . \nabla C_{1}+C_{1} g_{1}(T)=0 \\
\partial_{t} C_{2}-d_{2} \Delta C_{2}+u . \nabla C_{2}+C_{2} g_{2}(T)-C_{1} g_{1}(T)=0 \\
\partial_{t} u-\nu \Delta u+(u \nabla) u+\nabla p=f(T) \\
\text { div } u=0
\end{array}\right.
$$

where the unknown factors are speed $u$, the pressure $p$, the temperature $T$, the concentrations $C_{1}, C_{2}$ of species $A$ and $B$ respectively; the coefficients $\nu, \lambda, d_{1}$ and $d_{2}$ are assumed to be a positive constants (physically, $d_{i}$ indicates the diffusion of the concentration $C_{i}(\mathrm{i}=1,2), \lambda$ the thermal diffusion and $\nu$ the viscosity of the fluid). The datas are a regular function $f$ of $\mathbb{R}$ to $\mathbb{R}^{d}$ (typically, the function $f$ is a gravity force proportional to the variations of density, therefore dependents on the temperature) and an other regular function $g_{i}\left(\mathrm{i}=1,2\right.$ ) of $\mathbb{R}$ to $\mathbb{R}^{d}$ (typically, the function $g_{i}(\mathrm{i}=1,2)$ is the source term of the reaction depending on the temperature and also on energy).

The gradient, divergence and laplacian operators can be defined as following:

$$
\nabla v=\left(\frac{\partial v}{\partial x_{1}}, \frac{\partial v}{\partial x_{2}}, \ldots, \frac{\partial v}{\partial x_{d}}\right) \quad \operatorname{div} \overrightarrow{\mathbf{v}}=\sum_{i=1}^{d} \frac{\partial \mathbf{v}_{i}}{\partial x_{i}}, \quad \Delta v=\sum_{i=1}^{d} \frac{\partial^{2} v}{\partial^{2} x_{i}}
$$

The boundary conditions are of Dirichlet type for speed and Dirichlet-Neumann type for the temperature same as for the concentration. They are written:

$$
\left.T\right|_{\Gamma_{1}}=\left.C_{i}\right|_{\Gamma_{1}},\left.\quad \frac{\partial T}{\partial n}\right|_{\Gamma_{2}}=\left.\frac{\partial C_{i}}{\partial n}\right|_{\Gamma_{2}}=0, \text { and }\left.u\right|_{\partial \Omega}=0 \quad i=1,2,
$$

and the initial conditions are given by :

$$
\left.u\right|_{t=0}=u_{0},\left.\quad T\right|_{t=0}=T_{0}, \text { and }\left.C_{i}\right|_{t=0}=C_{i_{0}} \quad i=1,2,
$$

where $\Gamma_{1}$ and $\Gamma_{2}$ are disjoined opens parts of $\partial \Omega$ such that $\overline{\Gamma_{1}} \cup \overline{\Gamma_{2}}=\partial \Omega$.

It will be useful to note that for the following assumption:

$$
f(T)=\beta\left(T-T_{0}\right), \lambda=0, \alpha(t)=g \gamma \text { and } g_{i}(T)=k_{i} \exp \left(-\frac{E_{i}}{R T}\right)
$$

the system of equations $(P)$ give the chemical model which is known under the name of thermo-diffusif model [3]. Where $\beta$ is the coefficient of thermal expansion, $T_{0}$ the average temperature and $g$ is the gravity constant. In our model, we announce that the function $g_{i}$ is multiplied by the concentration $C_{i}$, therefore the reactions are considered of the first order; this functions are obtained by the Arrhenius law [18]. Here $E_{i}$ indicates the activation energy, $R$ the universal gas constant, $g$ indicates the constant of gravity, $\gamma$ is the ascending unit vector, $T_{0}$ the initial temperature of the product, the index $i$ denotes the step number of the reaction. The existence and uniqueness of the continued problem solution is studied in [12].

In the next section, we establish the existence and the uniqueness of fully discritized problem and study its stability. 


\section{Presentation of the fully discretized problem}

Before giving our discretized problem. First, we will give some assumption which is in good agreement with the physical background and present our functional framework.

Assume that :

$$
\left\{\begin{array}{l}
\text { The reals } \nu, \lambda d_{1}, d_{2} \text { and } \lambda \text { are strictly positives, } \\
\alpha \in W^{1, \infty}(\mathbb{R}), \\
g_{i} \in W^{1, \infty}(\mathbb{R}), \quad C_{g_{i}}=\left\|g_{i}^{\prime}\right\|_{L^{\infty}(\Omega)}, \text { and } g_{i} \geq 0, \quad\left\|g_{i}\right\|_{L^{\infty}(\Omega)}=1, \quad i=1,2 \\
f \in W^{1, \infty}(\mathbb{R}), \quad f(0)=0, \\
\forall\left(T_{1}, T_{2}\right) \in\left(H_{0, \Gamma_{1}}^{1}(\Omega)\right)^{2}, \quad\left\|f\left(T_{1}\right)-f\left(T_{2}\right)\right\|_{L^{2}(\Omega)} \leq\left\|\nabla\left(T_{1}-T_{2}\right)\right\|_{L^{2}(\Omega)} .
\end{array}\right.
$$

We specify now the functional framework in which is carried out our analysis of the problem. The speeds space $V$ defined by:

$$
V=\left\{u \in\left(H_{0}^{1}(\Omega)\right)^{d} ; \text { divu }=0 \text { in } \Omega\right\} .
$$

The temperatures and the concentrations space is $H_{0, \Gamma_{1}}^{1}(\Omega)=\left\{v \in H^{1}(\Omega) /\left.v\right|_{\Gamma_{1}}=0\right\}$.

We introduce now the constant of Friedrichs - Poincaré related to the domain geometry:

$$
\rho=\sup _{u \in H_{0}^{1}(\Omega)} \frac{\|u\|_{L^{2}(\Omega)}}{\|\nabla u\|_{L^{2}(\Omega)}} .
$$

Throughout the paper, we often use the following notation:

For each $\zeta, \eta>0: \zeta \lesssim \eta \Leftrightarrow \exists C^{\star}>0: \zeta \leq C^{\star} \eta$; without further specification, we intend the constant $C^{\star}$ independent of the mesh-size and the solutions.

Now, we give our functional framework by introducing some spaces, next, we give our fully discretized problem. First, we introduce the spaces that we need for our studies :

For any value of the real parameter $h>0$, we consider three spaces $X_{h}, M_{h}$ and $W_{h}$ such as

$$
X_{h} \subset\left(H_{0}^{1}(\Omega)\right)^{d}, M_{h} \subset L_{0}^{2}(\Omega) \text { and } W_{h} \subset H_{0, \Gamma_{1}}^{1}(\Omega)
$$

we set

$$
V_{h}:=\left\{v_{h}, \quad \forall q_{h} \in M_{h}, \int_{\Omega} q_{h} d i v v_{h} d x=0\right\},
$$

and we assume that they satisfy the following conditions:

1. For any $0<\sigma \leq 1$, there exist a linear continuous operator $P_{h}$ from $H^{\sigma}(\Omega) \cap L_{0}^{2}(\Omega)$ onto $M_{h}$ such as

$$
\forall q \in H^{\sigma}(\Omega) \cap L_{0}^{2}(\Omega),\left\|q-P_{h} q\right\|_{0, \Omega} \lesssim h^{\sigma}|q|_{\sigma, \Omega},
$$

2. For all $0<\sigma \leq 1$, there exist a linear continuous operator $\mathcal{I}_{h}$ from $\left(H^{1+\sigma}(\Omega)\right)^{d} \cap\left(H_{0}^{1}(\Omega)\right)^{d}$ onto $X_{h}$ such as

$$
\forall u \in\left(H^{1+\sigma}(\Omega)\right)^{d} \cap\left(H_{0}^{1}(\Omega)\right)^{d}, \quad\left\|u-\mathcal{I}_{h} u\right\|_{1, \Omega} \lesssim h^{\sigma}|u|_{1+\sigma, \Omega}
$$

3. there exist a constant $\beta$ independent of $h$, such as

$$
\forall q_{h} \in M_{h}, \exists v_{h} \in X_{h}, \quad \text { such as }\left(\operatorname{div}_{h}, q_{h}\right)_{0, \Omega} \geq \beta\left\|q_{h}\right\|_{0, \Omega}\left\|v_{h}\right\|_{1, \Omega} .
$$

4. For all $0<\sigma \leq 1$, there exist a linear continuous operator $i_{h}$ from $H^{1+\sigma}(\Omega) \cap H_{0}^{1}(\Omega)$ onto $W_{h}$ such as

$$
\forall T \in H^{1+\sigma}(\Omega) \cap H_{0}^{1}(\Omega), \quad\left\|T-i_{h} T\right\|_{1, \Omega} \lesssim h^{\sigma}|T|_{1+\sigma, \Omega} .
$$

We give now some examples of such couple of spaces verifying the last conditions [8]. We assume that the open $\Omega$ is polyhedric and we assume a regular triangulations family $\tau_{h}$ of $\Omega$, where for all $h$ the triangulation $\tau_{h}$ is $d$-simplexes set of diameters bounded above by $h$. For all $K$ of $\tau_{h}$, we define by $P_{k}(K)$ the polynomial space of total degree $k$ on $K$, where $k$ is a strictly positive real.

Let the space $W_{h}$ defined as following:

$$
W_{h}=\left\{T_{h} \in C^{0}(\Omega) \cap H_{0}^{1}(\Omega) ; \forall K \in \tau_{h} ; T_{h \mid K} \in P_{1}(K)\right\} .
$$

For the spaces $X_{h}$ and $M_{h}$ we have the following examples: 
Example 1 In dimension $d=2$, we set

$$
\begin{gathered}
X_{h}=\left\{v_{h} \in\left(C_{0}(\Omega)\right)^{2} \cap\left(H_{0}^{1}(\Omega)\right)^{2}, \forall K \in \tau_{h} ; v_{h \mid K} \in\left(P_{2}(K)\right)^{2}\right\}, \\
M_{h}=\left\{q_{h} \in L_{0}^{2}(\Omega), \forall K \in \tau_{h} ; q_{h \mid K} \in P_{0}(K)\right\},
\end{gathered}
$$

Example 2 In dimension $d=2$, we set

$$
\begin{gathered}
X_{h}=\left\{v_{h} \in\left(C_{0}(\Omega)\right)^{2} \cap\left(H_{0}^{1}(\Omega)\right)^{2}, \forall K \in \tau_{h} ; v_{h \mid K} \in\left(P_{2}(K)\right)^{2}\right\}, \\
M_{h}=\left\{q_{h} \in L_{0}^{2}(\Omega) \cup C_{0}(\Omega), \forall K \in \tau_{h} ; q_{h \mid K} \in P_{1}(K)\right\},
\end{gathered}
$$

Example 3 For all $T$ of $\tau_{h}$ of vertices $a_{i}, 1 \leq i \leq d+1$, we note by $\lambda_{i}$ the barycentric coordinate associated to the vertices $a_{i}$ and by $n_{i}$ the normal vector on the face not containing $a_{i}$. We set $P_{T}$ the space engendered by the polynoms of $\left(P_{1}(T)\right)^{d}$ and by the functions

$$
p_{i}=\left\{\prod_{j=1, j \neq i}^{d+1} \lambda_{i} n_{i}, 1 \leq i \leq d+1\right\} .
$$

We set then

$$
\begin{gathered}
X_{h}=\left\{v_{h} \in\left(C_{0}(\Omega)\right)^{d} \cap\left(H_{0}^{1}(\Omega)\right)^{d}, \forall K \in \tau_{h} ; v_{h \mid K} \in P_{K}\right\}, \\
M_{h}=\left\{q_{h} \in L_{0}^{2}(\Omega) \cup C_{0}(\Omega), \forall K \in \tau_{h} ; q_{h \mid K} \in P_{1}(K)\right\},
\end{gathered}
$$

In the three examples above, the assumptions $(1)-(3)$ are satisfied and the constant $\beta$ is independent of $h[7,10]$.

Let the following spaces:

$$
X=\left(H_{0, \Gamma_{1}}^{1}(\Omega)\right)^{d}, W=H_{0, \Gamma_{1}}^{1}(\Omega), \quad M=L_{0}^{2}(\Omega), \quad V=\{v \in X, \operatorname{div} v=0\} .
$$

and we introduce the forms defined by:

for all $(u, v, w) \in X^{3},(T, \phi, C, \psi) \in W^{4}, p \in M$,

$$
\begin{gathered}
a_{1}(u, v, w)=\frac{1}{2}\left(\int_{\Omega}(u \nabla) v w d x-\int_{\Omega}(u \nabla) w v d x\right) \\
a_{2}(u, T, \phi)=\frac{1}{2}\left(\int_{\Omega}(u \nabla) T \phi d x-\int_{\Omega}(u \nabla) \phi T d x\right), \\
d(T, \psi)=\int_{\Omega} \nabla T \nabla \psi d x, \quad b(p, v)=\int_{\Omega} p d i v v d x
\end{gathered}
$$

and:

$$
k_{i}(C, T, \psi)=\int_{\Omega} C g_{i}(T) \psi d x .
$$

In order to give the fully discretized problem, we will use in time the finite difference method with implicit scheme.

Let $k=\Delta t$. We consider the following fully discretized schema problem:

For $C_{1_{h}}^{n}, C_{2_{h}}^{n}, T_{h}^{n}, u_{h}^{n}$ known, find $C_{i_{h}}^{n+1} \in W_{h}(i=1,2), T_{h}^{n+1} \in W_{h}, u_{h}^{n+1} \in X_{h}$ and $p_{h}^{n+1} \in M_{h}$ such that:

$$
\left(P_{h}^{n}\right)\left\{\begin{array}{c}
\forall \phi_{h} \in W_{h}, \quad\left(C_{1_{h}}^{n+1}, \phi_{h}\right)_{0, \Omega}+k d_{1} d\left(C_{1_{h}}^{n+1}, \phi_{h}\right)+k a_{1}\left(u_{h}^{n}, C_{1_{h}}^{n+1}, \phi_{h}\right) \\
\quad+k K\left(C_{1_{h}}^{n+1}, T_{h}^{n}, \phi_{h}\right)=\left(C_{1_{h}}^{n}, \phi_{h}\right)_{0, \Omega}, \\
\forall \phi_{h} \in W_{h}, \quad\left(C_{2_{h}}^{n+1}, \phi_{h}\right)_{0, \Omega}+k d_{2} d\left(C_{2_{h}}^{n+1}, \phi_{h}\right)+k a_{1}\left(u_{h}^{n}, C_{2_{h}}^{n+1}, \phi_{h}\right) \\
\quad+k K_{2}\left(C_{2_{h}}^{n+1}, T_{h}^{n}, \phi_{2_{h}}\right)-k K_{1}\left(C_{1_{h}}^{n+1}, T_{h}^{n}, \phi_{2_{h}}\right)=\left(C_{2_{h}}^{n}, \phi_{2_{h}}\right)_{0, \Omega}, \\
\forall \phi_{h} \in W_{h}, \quad\left(T_{h}^{n+1}, \phi_{h}\right)_{0, \Omega}+k \lambda d\left(T_{h}^{n+1}, \phi_{h}\right)+k a_{1}\left(u_{h}^{n}, T_{h}^{n+1}, \phi_{h}\right) \\
\quad-k K_{1}\left(C_{1_{h}}^{n+1}, T_{h}^{n}, \phi_{h}\right)-k K_{2}\left(C_{2_{h}}^{n+1}, T_{h}^{n}, \phi_{h}\right)=\left(T_{h}^{n}, \phi_{h}\right)_{0, \Omega}, \\
\forall v_{h} \in X_{h}, \quad\left(u_{h}^{n+1}, v_{h}\right)_{0, \Omega}+k \mu d\left(u_{h}^{n+1}, v_{h}\right)+k a_{1}\left(u_{h}^{n}, u_{h}^{n+1}, v_{h}\right)-k b\left(p_{h}^{n+1}, v_{h}\right)= \\
\quad k \alpha\left(t^{n+1}\right)\left(f\left(T_{h}^{n+1}\right), v_{h}\right)_{0, \Omega}+\left(u_{h}^{n}, v_{h}\right)_{0, \Omega} \\
\forall q_{h} \in M_{h}, \quad b\left(q_{h}, u_{h}^{n+1}\right)=0 .
\end{array}\right.
$$

As usual, we consider that the couple of spaces $\left(X_{h}, M_{h}\right)$ is compatible. By classical arguments [7, 10], we have the following lemma: 
Lemma 3.1. For all $n \in N^{*}$, the problem admits a unique solution

$$
\left(u_{h}^{n}, p_{h}^{n}, T_{h}^{n}, C_{1_{h}}^{n}, C_{2_{h}}^{n}\right) \in X_{h} \times M_{h} \times W_{h}^{3} .
$$

In the sequel of this paper, for each $n$ positive integer, we denoted by $C_{1_{h}}^{n}, C_{2_{h}}^{n}, T_{h}^{n}, u_{h}^{n}$ and $p_{h}^{n}$ the discret solution of the problem $\left(P_{h}^{n}\right)$.

\section{Study of stability}

The main result of this section is to prove stability of our fully disretized schema. To prove that we need some lemmas. First we have:

Lemma 4.1. For all $n \in N^{*}$, we have:

$$
\left\|C_{1_{h}}^{n}\right\|_{0, \Omega}^{2}+\sum_{i=0}^{n-1}\left\|C_{1_{h}}^{i+1}-C_{1_{h}}^{i}\right\|_{0, \Omega}^{2}+2 k d_{1} \sum_{i=1}^{n}\left\|C_{1_{h}}^{i}\right\|_{1, \Omega}^{2} \leq\left\|C_{1_{h}}^{0}\right\|_{0, \Omega}^{2} .
$$

Proof. First of all, let us notice that:

$$
a_{1}\left(u_{1_{h}}^{n}, C_{1_{h}}^{n+1}, C_{1_{h}}^{n+1}\right)=0 \quad \text { and } K\left(C_{1_{h}}^{n+1}, T_{h}^{n}, C_{1_{h}}^{n+1}\right) \geq 0 .
$$

Consequently, by choosing $C_{1_{h}}^{n+1}$ as test function in the first equation of the problem $\left(P_{h}^{n}\right)$ and by using the identity $a(a-b)=\frac{1}{2}\left(a^{2}-b^{2}+(a-b)^{2}\right)$, we have:

$$
\left\|C_{1_{h}}^{n+1}\right\|_{0, \Omega}^{2}+\left\|C_{1_{h}}^{n+1}-C_{1_{h}}^{n}\right\|_{0, \Omega}^{2}+2 k d_{1}\left\|C_{1_{h}}^{n+1}\right\|_{1, \Omega}^{2} \leq\left\|C_{1_{h}}^{n}\right\|_{0, \Omega}^{2} .
$$

By summing over the time, we obtain:

$$
\left\|C_{1_{h}}^{n+1}\right\|_{0, \Omega}^{2}+\sum_{i=0}^{n}\left\|C_{1_{h}}^{i+1}-C_{1_{h}}^{i}\right\|_{0, \Omega}^{2}+2 k d_{1} \sum_{i=1}^{n+1}\left\|C_{1_{h}}^{i}\right\|_{1, \Omega}^{2} \leq\left\|C_{1_{h}}^{0}\right\|_{0, \Omega}^{2}
$$

We have also the following:

Lemma 4.2. For all $n \in N^{*}$, we have:

$$
\left\|C_{2_{h}}^{n}\right\|_{0, \Omega}^{2}+\sum_{i=0}^{n-1}\left\|C_{2_{h}}^{i+1}-C_{2_{h}}^{i}\right\|_{0, \Omega}^{2}+d_{2} k \sum_{i=1}^{n}\left\|C_{2_{h}}^{i}\right\|_{1, \Omega}^{2} \leq\left\|C_{2_{h}}^{0}\right\|_{0, \Omega}^{2}+\frac{\rho^{4}}{2 d_{1} d_{2}}\left\|C_{1_{h}}^{0}\right\|_{0, \Omega}^{2} .
$$

Proof. By choosing $C_{2_{h}}^{n+1}$ as test function in the second equation of the problem $\left(P_{h}^{n}\right)$, while noticing that : $a_{1}\left(u_{h}^{n}, C_{2_{h}}^{n+1}, C_{2_{h}}^{n+1}\right)=0, K_{2}\left(C_{2_{h}}^{n+1}, T_{h}^{n}, C_{2_{h}}^{n+1}\right) \leq 0$ and by using the identity $a(a-b)=\frac{1}{2}\left(a^{2}-b^{2}+(a-b)^{2}\right)$, we have:

However:

$$
\left\|C_{2_{h}}^{n+1}\right\|_{0, \Omega}^{2}+\left\|C_{2_{h}}^{n+1}-C_{2_{h}}^{n}\right\|_{0, \Omega}^{2}+2 k d_{2}\left\|C_{2_{h}}^{n+1}\right\|_{1, \Omega}^{2} \leq 2 k K_{1}\left(C_{1_{h}}^{n+1}, T_{h}^{n}, C_{2_{h}}^{n+1}\right)+\left\|C_{2_{h}}^{n}\right\|_{0, \Omega}^{2} .
$$

$$
K_{1}\left(C_{1_{h}}^{n+1}, T_{h}^{n}, C_{2_{h}}^{n+1}\right) \leq \frac{\rho^{4}}{2 d_{2}}\left\|C_{1_{h}}^{n+1}\right\|_{1, \Omega}^{2}+\frac{d_{2}}{2}\left\|C_{2_{h}}^{n+1}\right\|_{1, \Omega}^{2}
$$

Therefore:

$$
\left\|C_{2_{h}}^{n+1}\right\|_{0, \Omega}^{2}+\left\|C_{2_{h}}^{n+1}-C_{2_{h}}^{n}\right\|_{0, \Omega}^{2}+k d_{2}\left\|C_{2_{h}}^{n+1}\right\|_{1, \Omega}^{2} \leq\left\|C_{2_{h}}^{n}\right\|_{0, \Omega}^{2}+k \frac{\rho^{4}}{d_{2}}\left\|C_{1_{h}}^{n+1}\right\|_{1, \Omega}^{2} .
$$

By summing over the steps time and by using Lemma 4.1, we deduce the following estimate:

$$
\left\|C_{2_{h}}^{n+1}\right\|_{0, \Omega}^{2}+\sum_{i=0}^{n}\left\|C_{2_{h}}^{i+1}-C_{2_{h}}^{i}\right\|_{0, \Omega}^{2}+k d_{2} \sum_{i=1}^{n+1}\left\|C_{2_{h}}^{i}\right\|_{1, \Omega}^{2} \leq\left\|C_{2_{h}}^{0}\right\|_{0, \Omega}^{2}+\frac{\rho^{4}}{2 d_{1} d_{2}}\left\|C_{1_{h}}^{0}\right\|_{0, \Omega}^{2} .
$$

We have also the following:

Lemma 4.3. For all $n \in N^{*}$, we have:

$$
\left\|T_{h}^{n}\right\|_{0, \Omega}^{2}+\sum_{i=0}^{n-1}\left\|T_{h}^{i+1}-T_{h}^{i}\right\|_{0, \Omega}^{2}+\lambda k \sum_{i=1}^{n}\left\|T_{h}^{i}\right\|_{1, \Omega}^{2} \leq\left\|T_{h}^{0}\right\|_{0, \Omega}^{2}+\frac{\rho^{4}}{\lambda d_{1}}\left(1+\frac{\rho^{4}}{d_{2}^{2}}\right)\left\|C_{1_{h}}^{0}\right\|_{0, \Omega}^{2}+\frac{2 \rho^{4}}{\lambda d_{2}}\left\|C_{2_{h}}^{0}\right\|_{0, \Omega}^{2} .
$$


Proof. By choosing $T_{h}^{n+1}$ as test function in the second equation of the problem $\left(P_{h}^{n}\right)$, while noticing that : $a_{1}\left(u_{h}^{n}, T_{h}^{n+1}, T_{h}^{n+1}\right)=0$ and by using the identity $a(a-b)=\frac{1}{2}\left(a^{2}-b^{2}+(a-b)^{2}\right)$, we have:

$$
\begin{gathered}
\left\|T_{h}^{n+1}\right\|_{0, \Omega}^{2}+\left\|T_{h}^{n+1}-T_{h}^{n}\right\|_{0, \Omega}^{2}+2 k \lambda\left\|T_{h}^{n+1}\right\|_{1, \Omega}^{2} \leq 2 k K_{1}\left(C_{1_{h}}^{n+1}, T_{h}^{n}, T_{h}^{n+1}\right)+ \\
2 k K_{2}\left(C_{2_{h}}^{n+1}, T_{h}^{n}, T_{h}^{n+1}\right)+\left\|T_{h}^{n}\right\|_{0, \Omega}^{2} .
\end{gathered}
$$

However:

$$
K\left(C_{1_{h}}^{n+1}, T_{h}^{n}, T_{h}^{n+1}\right) \leq \frac{\rho^{4}}{\lambda}\left\|C_{1_{h}}^{n+1}\right\|_{1, \Omega}^{2}+\frac{\lambda}{4}\left\|T_{h}^{n+1}\right\|_{1, \Omega}^{2}
$$

Therefore:

$$
\left\|T_{h}^{n+1}\right\|_{0, \Omega}^{2}+\left\|T_{h}^{n+1}-T_{h}^{n}\right\|_{0, \Omega}^{2}+k \lambda\left\|T_{h}^{n+1}\right\|_{1, \Omega}^{2} \leq\left\|T_{h}^{n}\right\|_{0, \Omega}^{2}+k \frac{2 \rho^{4}}{\lambda}\left(\left\|C_{1_{h}}^{n+1}\right\|_{1, \Omega}^{2}+\left\|C_{2_{h}}^{n+1}\right\|_{1, \Omega}^{2}\right) .
$$

By summing over the steps time and by using Lemma 4.1 and Lemma 4.2 , we deduce the following estimate:

$$
\begin{aligned}
\left\|T_{h}^{n+1}\right\|_{0, \Omega}^{2} & +\sum_{i=0}^{n}\left\|T_{h}^{i+1}-T_{h}^{i}\right\|_{0, \Omega}^{2}+k \lambda \sum_{i=1}^{n+1}\left\|T_{h}^{i}\right\|_{1, \Omega}^{2} \leq\left\|T_{h}^{0}\right\|_{0, \Omega}^{2} \\
& +\frac{2 k \rho^{4}}{\lambda}\left(\frac{1}{2 k d_{1}}\left\|C_{1_{h}}^{0}\right\|+\frac{\rho^{4}}{2 k d_{1} d_{1}^{2}}\left\|C_{1_{h}}^{0}\right\|+\frac{1}{k d_{2}}\left\|C_{2_{h}}^{0}\right\|_{0, \Omega}^{2}\right)
\end{aligned}
$$

Also, we have the following:

Lemma 4.4. For all $n \in N^{*}$, we have:

$$
\left\{\begin{aligned}
\left\|u_{h}^{n}\right\|_{0, \Omega}^{2}+\sum_{i=0}^{n-1}\left\|u_{h}^{i+1}-u_{h}^{i}\right\|_{0, \Omega}^{2}+k \nu \sum_{i=1}^{n}\left\|u_{h}^{i}\right\|_{1, \Omega} \leq & \frac{\alpha^{2} \rho^{2}}{\lambda \nu}\left(\left\|T_{h}^{0}\right\|_{0, \Omega}^{2}+\frac{\rho^{4}}{\lambda d_{1}}\left(1+\frac{\rho^{4}}{d_{2}^{2}}\right)\left\|C_{1_{h}}^{0}\right\|_{0, \Omega}^{2}\right. \\
& \left.+\frac{2 \rho^{4}}{\lambda d_{2}}\left\|C_{2_{h}}^{0}\right\|_{0, \Omega}^{2}\right)+\left\|u_{h}^{0}\right\|_{0, \Omega}^{2} .
\end{aligned}\right.
$$

Proof. By choosing $u_{h}^{n+1} \in V_{h}$ as test function in the third equation of the problem $\left(P_{h}^{n}\right)$, while noticing that $a_{1}\left(u_{h}^{n}, u_{h}^{n+1}, u_{h}^{n+1}\right)=0$ and by re-using the identity $a(a-b)=\frac{1}{2}\left(a^{2}-b^{2}+(a-b)^{2}\right)$, we have:

$$
\left\|u_{h}^{n+1}\right\|_{0, \Omega}^{2}+\left\|u_{h}^{n+1}-u_{h}^{n}\right\|_{0, \Omega}^{2}+2 k \nu\left\|u_{h}^{n+1}\right\|_{1, \Omega}^{2}=2 k \alpha\left(t^{n+1}\right)\left(f\left(T_{h}^{n+1}\right), u_{h}^{n+1}\right)+\left\|u_{h}^{n}\right\|_{0, \Omega}^{2}
$$

However:

$$
2 k \alpha\left(t^{n+1}\right)\left(f\left(T_{h}^{n+1}, u_{h}^{n+1}\right) \leq k \nu\left\|u_{h}^{n+1}\right\|_{1, \Omega}^{2}+\frac{\alpha^{2} \rho^{2}}{\nu} k\left\|T_{h}^{n+1}\right\|_{1, \Omega}^{2}\right.
$$

Therefore by using Lemma 4.1, Lemma 4.2 and Lemma 4.3, we deduce that:

$$
\begin{aligned}
\left\|u_{h}^{n}\right\|_{0, \Omega}^{2}+\sum_{i=0}^{n-1}\left\|u_{h}^{i+1}-u_{h}^{i}\right\|_{0, \Omega}^{2}+k \nu \sum_{i=1}^{n}\left|u_{h}^{i}\right|_{1, \Omega} \leq & \frac{\alpha^{2} \rho^{2}}{\lambda \nu}\left(\left\|T_{h}^{0}\right\|_{0, \Omega}^{2}+\frac{\rho^{4}}{\lambda d_{1}}\left(1+\frac{\rho^{4}}{d_{2}^{2}}\right)\left\|C_{1_{h}}^{0}\right\|_{0, \Omega}^{2}\right. \\
& \left.+\frac{2 \rho^{4}}{\lambda d_{2}}\left\|C_{2_{h}}^{0}\right\|_{0, \Omega}^{2}\right)+\left\|u_{h}^{0}\right\|_{0, \Omega}^{2} .
\end{aligned}
$$

Now, using the Lemmas 4.1, 4.2, 4.3 and 4.4, we easily deduce stability result:

Theorem 4.1. For all $n$ positive integer the fully discitized problem $\left(P_{h}^{n}\right)$ is stable.

In the next section, we obtain some error estimates at the same time on speed, the pressure, the temperature and the concentrations.

\section{Error stimates}

We consider the operators $R_{h}$ and $r_{h}$ respectively definite from $\left(H_{0}^{1}(\Omega)\right)^{d}$ and $H_{0, \Gamma_{1}}^{1}(\Omega)$ onto $X_{h}$ and $W_{h}$ by:

$$
\forall u \in\left(H_{0}^{1}(\Omega)\right)^{d}, \forall v_{h} \in X_{h}, \quad \int_{\Omega} \nabla\left(R_{h} u-u\right) . \nabla v_{h}=0,
$$

and

$$
\forall s \in H_{0, \Gamma_{1}}^{1}(\Omega), \forall \phi_{h} \in W_{h}, \quad \int_{\Omega} \nabla\left(r_{h} s-s\right) . \nabla \phi_{h}=0
$$


We remind that:

$$
\forall u \in\left(H_{0}^{1}(\Omega)\right)^{d}, \quad\left\|\nabla R_{h} u\right\|_{1, \Omega} \leq\|\nabla u\|_{1, \Omega} .
$$

Moreover, if $u \in\left(H^{1+\sigma}(\Omega)\right)^{d}$, with $1<\sigma \leq 2$, we have [8]:

$$
\left\|u-R_{h} u\right\|_{1, \Omega} \lesssim h^{\sigma}\|u\|_{1+\sigma, \Omega} .
$$

We have also [8],

$$
\forall s \in\left(H_{0, \Gamma_{1}}^{1}(\Omega)\right)^{d}, \quad\left\|\nabla r_{h} s\right\|_{1, \Omega} \leq\|\nabla s\|_{1, \Omega} .
$$

Moreover, if $s \in\left(H^{1+\sigma}(\Omega)\right)^{d}$, with $1<\sigma \leq 2$, we have, [8]:

$$
\left\|r_{h} s-s\right\|_{1, \Omega} \lesssim h^{\sigma}\|s\|_{1+\sigma, \Omega} .
$$

In the following, we set:

$$
\zeta^{n}=R_{h} u^{n}-u_{h}^{n}, \eta^{n}=r_{h} T^{n}-T_{h}^{n} \text { and } \epsilon_{i}^{n}=r_{i_{h}} C^{n}-C_{i_{h}}^{n}, \quad(i=1,2)
$$

and

$$
\bar{\partial}_{t} \phi^{n}=\frac{\phi^{n}-\phi^{n-1}}{k}
$$

Finally, we set:

$$
\begin{gathered}
A=\|u\|_{L^{\infty}\left(0, t,\left(H^{1}(\Omega)\right)^{2}\right)}, M=\sup _{0 \leq k \leq N}\left\|u_{h}^{k}\right\|_{1, \Omega}, B=\|T\|_{L^{\infty}\left(0, t, H^{1}(\Omega)\right)}, \\
M_{c}=\max _{i=1,2}\left(\left\|C_{i}\right\|_{L^{\infty}\left(0, t, H^{1}(\Omega)\right)}\right), C_{g}=\|g\|_{L^{\infty}(\mathbb{R})} .
\end{gathered}
$$

In this section, our main theorem is the following:

Theorem 5.1. Assume that:

$$
N A<\nu+N M, \quad N B<\frac{\lambda}{2}, \quad N M_{C}<\frac{\lambda}{2}
$$

and

$$
16 M_{C}^{2} C_{g}^{2}<\min \left(\lambda, \frac{\lambda}{2 \rho^{2}}\right), \quad k \leq \frac{1}{4}, \quad w=\max \left(\frac{120}{7} k, \frac{304}{7} k M_{C}^{2} C_{g}^{2}\right)<1 .
$$

If the solution of the problem $(P)$ admits the following regularity:

$$
\left\{\begin{array}{l}
u \in W^{2, \infty}\left(0, t,\left(L^{2}(\Omega)\right)^{d}\right) \cap W^{1, \infty}\left(0, t,\left(H^{1}(\Omega)\right)^{d}\right) \cap L^{\infty}\left(0, t,\left(H^{1+\sigma}(\Omega)\right)^{d}\right), \\
p \in L^{\infty}\left(0, t, H^{\sigma}(\Omega)\right), \\
T, C_{1}, C_{2} \in W^{2, \infty}\left(0, t,\left(L^{2}(\Omega)\right)\right) \cap W^{1, \infty}\left(0, t, H^{1}(\Omega)\right) \cap L^{\infty}\left(0, t, H^{1+\sigma}(\Omega)\right),
\end{array}\right.
$$

then we have the following error estimates:

$$
\left\|u^{n}-u_{h}^{n}\right\|_{0, \Omega}^{2}+k\left\|\nabla\left(u^{n}-u_{h}^{n}\right)\right\|_{0, \Omega}^{2} \lesssim\left(h^{2 \sigma}+k^{2}\right)\left(k n+k^{2} n^{2}\right)
$$

for the velocity,

$$
\left\|p^{n}-p_{h}^{n}\right\|_{0, \Omega}^{2} \lesssim\left(h^{2 \sigma}+k^{2}\right)\left(k n+k^{2} n^{2}+k^{3} n^{3}\right)
$$

for the pressure,

$$
\left\|T^{n}-T_{h}^{n}\right\|_{0, \Omega}^{2}+\frac{\lambda k}{1-w}\left\|\nabla\left(T^{n}-T_{h}^{n}\right)\right\|_{0, \Omega}^{2} \lesssim\left(h^{2 \sigma}+k^{2}\right)\left(k n+k^{2} n^{2}+k^{3} n^{3}\right)
$$

for the temperature and

$$
\left\|C_{i}^{n}-C_{i_{h}}^{n}\right\|_{0, \Omega}^{2}+\frac{16}{7(1-w)} k\left(\lambda-3 \rho^{2}\right)\left\|\nabla\left(C_{i}^{n}-C_{i_{h}}^{n}\right)\right\|_{0, \Omega}^{2} \lesssim\left(h^{2 \sigma}+k^{2}\right)\left(k n+k^{2} n^{2}+k^{3} n^{3}\right),
$$

for the concentrations $(i=1,2)$.

In the sequel of this paper, we assume:

$$
\left\{\begin{array}{l}
u \in W^{2, \infty}\left(0, t,\left(L^{2}(\Omega)\right)^{d}\right) \cap W^{1, \infty}\left(0, t,\left(H^{1}(\Omega)\right)^{d}\right) \cap L^{\infty}\left(0, t,\left(H^{1+\sigma}(\Omega)\right)^{d}\right), \\
p \in L^{\infty}\left(0, t, H^{\sigma}(\Omega)\right) \\
T, C_{1}, C_{2} \in W^{2, \infty}\left(0, t,\left(L^{2}(\Omega)\right)\right) \cap W^{1, \infty}\left(0, t, H^{1}(\Omega)(\Omega)\right) \cap L^{\infty}\left(0, t, H^{1+\sigma}(\Omega)\right),
\end{array}\right.
$$

To prove the a priori estimate on the pressure, we need some lemmas, first we have: 
Lemma 5.1. For all $v_{h} \in V_{h}$ and $n \in N^{*}$, we have:

$$
\begin{aligned}
\left|a_{1}\left(u_{h}^{n-1}, u_{h}^{n}, v_{h}\right)-a_{1}\left(u^{n}, u^{n}, v_{h}\right)\right| \leq\left(N M\left\|u^{n}-u_{h}^{n}\right\|_{1, \Omega}+N A\left\|u^{n-1}-u_{h}^{n-1}\right\|_{1, \Omega}\right. \\
\\
\left.+N A k\|u\|_{\left.W^{1, \infty}\left(0, t,\left(H^{1}(\Omega)\right)^{2}\right)\right)}\right)\left|v_{h}\right|_{1, \Omega} .
\end{aligned}
$$

Proof. By the triangular inequality, we have:

$$
\begin{aligned}
& \left|a_{1}\left(u_{h}^{n-1}, u_{h}^{n}, v_{h}\right)-a_{1}\left(u^{n}, u^{n}, v_{h}\right)\right| \leq\left|a_{1}\left(u_{h}^{n-1}, u_{h}^{n}, v_{h}\right)-a_{1}\left(u_{h}^{n-1}, u^{n}, v_{h}\right)\right| \\
& +\mid a_{1}\left(u_{h}^{n-1}, u^{n}, v_{h}\right)-a_{1}\left(u^{n-1}, u^{n}, v_{h}|+| a_{1}\left(u^{n-1}, u^{n}, v_{h}\right)-a_{1}\left(u^{n}, u^{n}, v_{h}\right) \mid .\right.
\end{aligned}
$$

The proof of this lemma is obtained by using the three following inequalities:

$$
\begin{aligned}
& \left|a_{1}\left(u_{h}^{n-1}, u_{h}^{n}, v_{h}\right)-a_{1}\left(u_{h}^{n-1}, u^{n}, v_{h}\right)\right| \leq N M\left\|u_{h}^{n}-u^{n}\right\|_{1, \Omega}\left\|v_{h}\right\|_{1, \Omega}, \\
& \mid a_{1}\left(u_{h}^{n-1}, u^{n}, v_{h}\right)-a_{1}\left(u^{n-1}, u^{n}, v_{h} \mid \leq N A\left\|u_{h}^{n-1}-u^{n-1}\right\|_{1, \Omega}\left\|v_{h}\right\|_{1, \Omega},\right.
\end{aligned}
$$

and

$$
\left|a_{1}\left(u^{n-1}, u^{n}, v_{h}\right)-a_{1}\left(u^{n}, u^{n}, v_{h}\right)\right| \leq N A k\|u\|_{\left.W^{1, \infty}\left(0, t,\left(H^{1}(\Omega)\right)^{2}\right)\right)}\left\|v_{h}\right\|_{1, \Omega} .
$$

Again, we have the following:

Lemma 5.2. For all real $\theta_{0}$ strictly positive independently of $h$ and $k$, we have:

$$
\left\{\begin{aligned}
\left|a_{1}\left(u_{h}^{n-1}, u_{h}^{n}, \zeta^{n}\right)-a_{1}\left(u^{n}, u^{n}, \zeta^{n}\right)\right| \leq \quad & \left(\theta_{0} N M+N A\right)\left\|u^{n}-u_{h}^{n}\right\|_{1, \Omega}^{2} \\
& +N A\left\|u^{n-1}-u_{h}^{n-1}\right\|_{1, \Omega}^{2}+O\left(h^{2 \sigma}+k^{2}\right) .
\end{aligned}\right.
$$

Proof. By the triangular inequality, we have:

$$
\begin{aligned}
& \left|a_{1}\left(u_{h}^{n-1}, u_{h}^{n}, \zeta^{n}\right)-a_{1}\left(u^{n}, u^{n}, \zeta^{n}\right)\right| \leq\left|a_{1}\left(u_{h}^{n-1}, u_{h}^{n}, \zeta^{n}\right)-a_{1}\left(u_{h}^{n-1}, u^{n}, \zeta^{n}\right)\right| \\
& +\left|a_{1}\left(u_{h}^{n-1}-u^{n-1}, u^{n}, R_{h} u^{n}-u^{n}+u^{n}-u_{h}^{n}\right)\right|+\left|a_{1}\left(u^{n-1}-u^{n}, u^{n}, R_{h} u^{n}-u^{n}+u^{n}-u_{h}^{n}\right)\right| .
\end{aligned}
$$

We have also:

$$
\begin{aligned}
\left\|u^{n}-u^{n-1}\right\|_{1, \Omega} & \lesssim k\|u\|_{W^{1, \infty}\left(0, t,\left(H^{1}(\Omega)\right)^{d}\right)}, \\
\left\|u^{n}-R_{h} u^{n}\right\|_{1, \Omega} & \lesssim h^{\sigma}\|u\|_{L^{\infty}\left(0, t,\left(H^{1+\sigma}(\Omega)\right)^{d}\right)}
\end{aligned}
$$

However

$$
\begin{gathered}
\mid a_{1}\left(u_{h}^{n-1}, u_{h}^{n}-u^{n}, \zeta^{n}\right) \leq N M\left\|u_{h}^{n}-u^{n}\right\|_{1, \Omega}\left\|\zeta^{n}\right\|_{1, \Omega}, \\
\left|a_{1}\left(u_{h}^{n-1}-u^{n-1}, u^{n}, R_{h} u^{n}-u^{n}+u^{n}-u_{h}^{n}\right)\right| \leq N A\left\|u_{h}^{n-1}-u^{n-1}\right\|_{1, \Omega}\left\|R_{h} u^{n}-u^{n}+u^{n}-u_{h}^{n}\right\|_{1, \Omega},
\end{gathered}
$$

and

$$
\left|a_{1}\left(u^{n-1}-u^{n}, u^{n}, R_{h} u^{n}-u^{n}+u^{n}-u_{h}^{n}\right)\right| \leq N A\left\|u^{n-1}-u^{n}\right\|_{1, \Omega}\left\|R_{h} u^{n}-u^{n}+u^{n}-u_{h}^{n}\right\|_{1, \Omega} .
$$

Therefore by using the previous lemma, the last three inequalities and Young inequality, we obtain:

$$
\begin{aligned}
\left|a_{1}\left(u_{h}^{n-1}, u_{h}^{n}, \zeta^{n}\right)-a_{1}\left(u^{n}, u^{n}, \zeta^{n}\right)\right| \leq \quad & \left(\theta_{0} N M+N A\right)\left\|u^{n}-u_{h}^{n}\right\|_{1, \Omega}^{2} \\
& +N A\left\|u^{n-1}-u_{h}^{n-1}\right\|_{1, \Omega}^{2}+O\left(h^{2 \sigma}+k^{2}\right) .
\end{aligned}
$$

We have again the following technical estimate:

Lemma 5.3. For $n$ positive integer, we have:

$$
\begin{array}{r}
\left\|\partial_{t} u^{n}-\bar{\partial}_{t} u_{h}^{n}\right\|_{-1, \Omega} \lesssim \quad\left(h^{\sigma}+k\right)+\rho(\nu+N M)\left\|u^{n}-u_{h}^{n}\right\|_{1, \Omega} \\
+\rho N A\left\|u^{n-1}-u_{h}^{n-1}\right\|_{1, \Omega}+\rho^{2} \alpha\left\|T^{n}-T_{h}^{n}\right\|_{1, \Omega}
\end{array}
$$


Proof. First of all, we have:

$$
\begin{aligned}
\left\|\partial_{t} u^{n}-\bar{\partial}_{t} u_{h}^{n}\right\|_{-1, \Omega} & \leq\left\|\partial_{t} u^{n}-\rho_{h} \partial_{t} u^{n}\right\|_{-1, \Omega}+\left\|\rho_{h} \partial_{t} u^{n}-\bar{\partial}_{t} u_{h}^{n}\right\|_{-1, \Omega} \\
& \leq\left\|\partial_{t} u^{n}-\rho_{h} \partial_{t} u^{n}\right\|_{-1, \Omega}+\sup _{v \in V} \frac{\left(\rho_{h} \partial_{t} u^{n}-\bar{\partial}_{t} u_{h}^{n}, v\right)_{0, \Omega}}{\|v\|_{1, \Omega}}
\end{aligned}
$$

However, for all $v_{h} \in V_{h}$, we have:

$$
\begin{aligned}
\left(\rho_{h} \partial_{t} u^{n}-\bar{\partial}_{t} u_{h}^{n}, v_{h}\right)_{0, \Omega}= & \left(\partial_{t} u^{n}, v_{h}\right)_{0, \Omega}-\left(\bar{\partial}_{t} u_{h}^{n}, v_{h}\right)_{0, \Omega} \\
= & -\nu d\left(u^{n}-u_{h}^{n}, v_{h}\right)+a_{1}\left(u_{h}^{n-1}, u_{h}^{n}, v_{h}\right)-a_{1}\left(u^{n}, u^{n}, v_{h}\right) \\
& +b\left(p^{n}-p_{h}^{n}, v_{h}\right)+\alpha\left(t^{n}\right)\left(f\left(T^{n}\right)-f\left(T_{h}^{n}\right), v_{h}\right) .
\end{aligned}
$$

On the one hand, we have:

$$
b\left(p^{n}-p_{h}^{n}, v_{h}\right)=b\left(p^{n}-P_{h} p^{n}, v_{h}\right)
$$

Therefore:

$$
b\left(p^{n}-p_{h}^{n}, v_{h}\right) \leq\left\|p^{n}-P_{h} p^{n}\right\|_{0, \Omega}\left\|v_{h}\right\|_{1, \Omega}
$$

then

$$
b\left(p^{n}-p_{h}^{n}, v_{h}\right) \lesssim h^{\sigma}\|p\|_{L^{\infty}\left(0, t, H^{\sigma}(\Omega)\right)}\left\|v_{h}\right\|_{1, \Omega} .
$$

On the other hand, by using the following estimates:

$$
\nu\left|d\left(u^{n}-u_{h}^{n}, v_{h}\right)\right| \leq \nu\left\|u^{n}-u_{h}^{n}\right\|_{1, \Omega}\left\|v_{h}\right\|_{1, \Omega},
$$

and

$$
\left|\alpha\left(t^{n}\right)\left(f\left(T^{n}\right)-f\left(T_{h}^{n}\right), v_{h}\right)\right| \leq \rho \alpha\left\|T^{n}-T_{h}^{n}\right\|_{1, \Omega}\left\|v_{h}\right\|_{1, \Omega} .
$$

By using the previous lemmas we have the following, which prove our lemma:

$$
\sup _{v_{h} \in V_{h}} \frac{\left(\rho_{h} \partial_{t} u^{n}-\bar{\partial}_{t} u_{h}^{n}, v_{h}\right)_{0, \Omega}}{\left\|v_{h}\right\|_{1, \Omega}} \lesssim \begin{gathered}
\left(h^{\sigma}+k\right)+(\nu+N M)\left\|u^{n}-u_{h}^{n}\right\|_{1, \Omega} \\
+N A\left\|u^{n-1}-u_{h}^{n-1}\right\|_{1, \Omega}+\rho \alpha\left\|T^{n}-T_{h}^{n}\right\|_{1, \Omega} .
\end{gathered}
$$

Finally, we are able to give the error estimate on the pressure:

Lemma 5.4. We have the following estimate:

$$
\begin{array}{r}
\left\|p^{n}-p_{h}^{n}\right\|_{0, \Omega} \lesssim \quad\left(h^{\sigma}+k\right)+\frac{1}{\beta}(1+\rho)(\nu+N M)\left\|\nabla\left(u^{n}-u_{h}^{n}\right)\right\|_{0, \Omega} \\
+\frac{1+\rho}{\beta} N A\left\|\nabla\left(u^{n-1}-u_{h}^{n-1}\right)\right\|_{0, \Omega}+\frac{\alpha \rho}{\beta}(1+\rho)\left\|\nabla\left(T^{n}-T_{h}^{n}\right)\right\|_{0, \Omega} .
\end{array}
$$

Proof. First of all, we have:

$$
\begin{aligned}
\left\|p^{n}-p_{h}^{n}\right\|_{0, \Omega} & \leq\left\|p^{n}-P_{h} p^{n}\right\|_{0, \Omega}+\left\|P_{h} p^{n}-p_{h}^{n}\right\|_{0, \Omega} \\
& \lesssim h^{\sigma}\|p\|_{L^{\infty}\left(H^{\sigma}\right)}+\beta^{-1} \sup _{v_{h} \in X_{h}} \frac{b\left(P_{h} p^{n}-p_{h}^{n}, v_{h}\right)}{\left\|\nabla v_{h}\right\|_{0, \Omega}} \\
& \lesssim h^{\sigma}\|p\|_{L^{\infty}\left(H^{\sigma}\right)}+\beta^{-1} \sup _{v_{h} \in X_{h}} \frac{b\left(p^{n}-p_{h}^{n}, v_{h}\right)}{\left\|\nabla v_{h}\right\|_{0, \Omega}}
\end{aligned}
$$

We look for finding estimate on $b\left(p^{n}-p_{h}^{n}, v_{h}\right)$

However, we have:

$$
\begin{gathered}
b\left(p^{n}-p_{h}^{n}, v_{h}\right)=b\left(p^{n}, v_{h}\right)-b\left(p_{h}^{n}, v_{h}\right), \\
b\left(p^{n}, v_{h}\right)=\nu d\left(u^{n}, v_{h}\right)+a_{1}\left(u^{n}, u^{n}, v_{h}\right)-\alpha\left(t^{n}\right)\left(f\left(T_{h}^{n}\right), v_{h}\right)+\left(\partial_{t} u^{n}, v_{h}\right)
\end{gathered}
$$

and

$$
b\left(p_{h}^{n}, v_{h}\right)=\nu d\left(u_{h}^{n}, v_{h}\right)+a_{1}\left(u_{h}^{n-1}, u_{h}^{n}, v_{h}\right)-\alpha\left(t^{n}\right)\left(f\left(T_{h}^{n}\right), v_{h}\right)+\left(\bar{\partial}_{t} u_{h}^{n}, v_{h}\right)
$$

From where:

$$
\begin{aligned}
b\left(p^{n}-p_{h}^{n}, v_{h}\right)= & \left(\partial_{t} u^{n}-\bar{\partial}_{t} u_{h}^{n}, v_{h}\right)+\nu d\left(u^{n}-u_{h}^{n}, v_{h}\right) \\
& +\left(a_{1}\left(u^{n}, u^{n}, v_{h}\right)-a_{1}\left(u_{h}^{n-1}, u_{h}^{n}, v_{h}\right)\right) \\
& -\alpha\left(t^{n}\right)\left(f\left(T^{n}\right)-f\left(T_{h}^{n}\right), v_{h}\right)
\end{aligned}
$$


We obtain then:

$$
\begin{aligned}
b\left(p^{n}-p_{h}^{n}, v_{h}\right) & \lesssim\left\|\partial_{t} u^{n}-\bar{\partial}_{t} u_{h}^{n}\right\|_{-1, \Omega}\left\|\nabla v_{h}\right\|_{0, \Omega}+(\nu+N M)\left\|\nabla\left(u^{n}-u_{h}^{n}\right)\right\|_{0, \Omega}\left\|\nabla v_{h}\right\|_{0, \Omega} \\
& +k\left\|\nabla v_{h}\right\|_{0, \Omega}+N A\left\|\nabla\left(u^{n-1}-u_{h}^{n-1}\right)\right\|_{0, \Omega}\left\|\nabla v_{h}\right\|_{0, \Omega} \\
& +\alpha \rho\left\|\nabla\left(T^{n}-T_{h}^{n}\right)\right\|_{0, \Omega}\left\|\nabla v_{h}\right\|_{0, \Omega},
\end{aligned}
$$

therefore:

$$
\begin{aligned}
b\left(p^{n}-p_{h}^{n}, v_{h}\right) & \lesssim\left(\left(h^{\sigma}+k\right)+(1+\rho)(\nu+N M)\left\|\nabla\left(u^{n}-u_{h}^{n}\right)_{0, \Omega}\right\|\right. \\
& \left.+(1+\rho)(N A)\left\|\nabla\left(u^{n-1}-u_{h}^{n-1}\right)\right\|_{0, \Omega}+(1+\rho) \alpha \rho\left\|\nabla\left(T^{n}-T_{h}^{n}\right)\right\|_{0, \Omega}\right)\left\|\nabla v_{h}\right\|_{0, \Omega} .
\end{aligned}
$$

From where, we deduce:

$$
\begin{aligned}
\left\|p^{n}-p_{h}^{n}\right\|_{0, \Omega} \lesssim & \left(h^{\sigma}+k\right)+\frac{1}{\beta}(1+\rho)(\nu+N M)\left\|\nabla\left(u^{n}-u_{h}^{n}\right)\right\|_{0, \Omega} \\
& +\frac{1+\rho}{\beta} N A\left\|\nabla\left(u^{n-1}-u_{h}^{n-1}\right)\right\|_{0, \Omega}+\frac{\alpha \rho}{\beta}(1+\rho)\left\|\nabla\left(T^{n}-T_{h}^{n}\right)\right\|_{0, \Omega} .
\end{aligned}
$$

Lemma 5.5. We have the following estimate:

$$
\left\|\bar{\partial}_{t} R_{h} u^{n}-\partial_{t} u^{n}\right\|_{0, \Omega} \lesssim k\|u\|_{W^{2, \infty}\left(0, t,\left(L^{2}(\Omega)\right)^{d}\right)}+h^{\sigma}\|u\|_{W^{1, \infty}\left(0, t,\left(H_{0}^{1}(\Omega)\right)^{d}\right)} .
$$

Proof. by triangular inequality we have:

$$
\left\|\bar{\partial}_{t} R_{h} u^{n}-\partial_{t} u^{n}\right\|_{0, \Omega} \leq\left\|\bar{\partial}_{t} R_{h} u^{n}-\bar{\partial}_{t} u^{n}\right\|_{0, \Omega}+\left\|\bar{\partial}_{t} u^{n}-\partial_{t} u^{n}\right\|_{0, \Omega}
$$

By using Taylor-Young formula with integral remainder, we obtain:

$$
\begin{aligned}
\left\|\bar{\partial}_{t} R_{h} u^{n}-\bar{\partial}_{t} u^{n}\right\|_{0, \Omega}+\left\|\bar{\partial}_{t} u^{n}-\partial_{t} u^{n}\right\|_{0, \Omega}= & \left\|\frac{1}{k} \int_{t^{n}-k}^{t^{n}}\left(R_{h} \partial_{t} u-\partial_{t} u\right) d s\right\|_{0, \Omega} \\
& +\left\|\int_{t^{n}-k}^{t^{n}}\left(s-t^{n-1}\right) \frac{\partial^{2}}{\partial t^{2}} u d s\right\|_{0, \Omega} .
\end{aligned}
$$

Therefore:

$$
\left\|\bar{\partial}_{t} R_{h} u^{n}-\partial_{t} u^{n}\right\|_{0, \Omega} \lesssim h^{\sigma}\|u\|_{\left.W^{1, \infty}\left(0, t, H_{0}^{1}(\Omega)\right)^{d}\right)}+k\|u\|_{\left.W^{2, \infty}\left(0, t, L^{2}(\Omega)\right)^{d}\right)} .
$$

Lemma 5.6. Assume that $N A<\nu+N M$, and $k \leq \frac{1}{4}$. Then:

$$
\left\|u^{n}-u_{h}^{n}\right\|_{0, \Omega}^{2}+k\left\|\nabla\left(u^{n}-u_{h}^{n}\right)\right\|_{0, \Omega}^{2} \lesssim\left(h^{2 \sigma}+k^{2}\right)+k \sum_{i=1}^{n}\left\|\nabla\left(T^{i}-T_{i}^{n}\right)\right\|_{0, \Omega}^{2} .
$$

Proof. For the proof of this lemma, we recall the following lemma, known as the Gronwal lemma:

Lemma 5.7. Let $a_{n}, b_{n}$ and $c_{n}$ three positive sequences, $c_{n}$ not decreasing sequence, Assume that

$$
a_{n}+b_{n} \leq c_{n}+\bar{\nu} \sum_{i=0}^{n-1} a_{i}, \quad \bar{\nu}>0
$$

and

$$
a_{0}+b_{0} \leq c_{0}
$$

We have the following result:

$$
a_{n} \leq c_{n} \exp (\bar{\nu} n)
$$

For $\xi^{n}=R_{h} u^{n}-u_{h}^{n} \in X_{h}$, we have:

$$
\left(\bar{\partial}_{t} \xi^{n}, \xi^{n}\right)+\nu d\left(u^{n}-u_{h}^{n}, u^{n}-u_{h}^{n}\right)=\nu d\left(u^{n}-u_{h}^{n}, u^{n}-u_{h}^{n}\right)+\left(\bar{\partial}_{t} R_{h} u^{n}, \xi^{n}\right)-\left(\bar{\partial}_{t} u_{h}^{n}, \xi^{n}\right) .
$$

However, if we choose $\xi^{n}$ as test function in the third equation of $\left(P_{h}^{n}\right)$, we obtain the following equation:

$$
\left(\bar{\partial}_{t} u_{h}^{n}, \xi^{n}\right)+\nu d\left(u_{h}^{n}, \xi^{n}\right)+a_{1}\left(u_{h}^{n-1}, u_{h}^{n}, \xi^{n}\right)-b\left(p_{h}^{n}, \xi^{n}\right)=\alpha\left(t^{n}\right)\left(f\left(T^{n}\right), \xi^{n}\right)
$$


By replacing the value of $\left(\bar{\partial}_{t} u_{h}^{n}, \xi^{n}\right)$, we obtain:

$$
\begin{aligned}
\left(\bar{\partial}_{t} \xi^{n}, \xi^{n}\right)+\nu d\left(u^{n}-u_{h}^{n}, u^{n}-u_{h}^{n}\right)= & \nu d\left(u^{n}-u_{h}^{n}, u^{n}-u_{h}^{n}\right)+\left(\bar{\partial}_{t} R_{h} u^{n}, \xi^{n}\right)+\nu d\left(u_{h}^{n}, \xi^{n}\right) \\
& +a_{1}\left(u_{h}^{n-1}, u_{h}^{n}, \xi^{n}\right)-b\left(p_{h}^{n}, \xi^{n}\right)-\alpha\left(t^{n}\right)\left(f\left(T_{h}^{n}\right), \xi^{n}\right),
\end{aligned}
$$

therefore:

$$
\begin{array}{r}
\left(\bar{\partial}_{t} \xi^{n}, \xi^{n}\right)+\nu d\left(u^{n}-u_{h}^{n}, u^{n}-u_{h}^{n}\right)=\nu d\left(u^{n}-u_{h}^{n}, u^{n}-u_{h}^{n}\right)+\left(\bar{\partial}_{t} R_{h} u^{n}-\partial_{t} u^{n}, \xi^{n}\right) \\
+\nu d\left(u_{h}^{n}, \xi^{n}\right)+a_{1}\left(u_{h}^{n-1}, u_{h}^{n}, \xi^{n}\right)-b\left(p_{h}^{n}, \xi^{n}\right)-\alpha\left(t^{n}\right)\left(f\left(T_{h}^{n}\right), \xi^{n}\right) \\
\quad-\nu d\left(u^{n}, \xi^{n}\right)-a_{1}\left(u^{n}, u^{n}, \xi^{n}\right)+b\left(p^{n}, \xi^{n}\right)+\alpha\left(t^{n}\right)\left(f\left(T^{n}\right), \xi^{n}\right),
\end{array}
$$

because:

$$
\left(\partial_{t} u^{n}, \xi^{n}\right)=-\nu d\left(u^{n}, \xi^{n}\right)-a_{1}\left(u^{n}, u^{n}, \xi^{n}\right)+b\left(p^{n}, \xi^{n}\right)+\alpha\left(t^{n}\right)\left(f\left(T^{n}\right), \xi^{n}\right) .
$$

Therefore:

$$
\begin{array}{r}
\left(\bar{\partial}_{t} \xi^{n}, \xi^{n}\right)+\nu d\left(u^{n}-u_{h}^{n}, u^{n}-u_{h}^{n}\right)= \\
+\left(a_{t} R_{h} u^{n}-\partial_{t} u^{n}, \xi^{n}\right)+\alpha\left(t^{n}\right)\left(f\left(T^{n}\right)-f\left(T_{h}^{n}\right), \xi^{n}\right) \\
+\nu\left(d\left(u^{n}-a_{1}\left(u^{n}, u^{n}, \xi^{n}\right)\right)+\left(b\left(p^{n}, \xi^{n}\right)-b\left(p_{h}^{n}, \xi^{n}\right)\right)+d\left(u_{h}^{n}, \xi^{n}\right)-d\left(u^{n}, \xi^{n}\right)\right) .
\end{array}
$$

we have:

$$
\begin{array}{rr}
\left.b\left(p^{n}, \xi^{n}\right)-b\left(p_{h}^{n}, \xi^{n}\right)\right)= & b\left(p^{n}, R_{h} u^{n}-u_{h}^{n}\right)-b\left(p_{h}^{n}, R_{h} u^{n}-u_{h}^{n}\right) \\
= & b\left(p^{n}-p_{h}^{n}, R_{h} u^{n}-u^{n}+u^{n}-u_{h}^{n}\right) \\
= & b\left(p^{n}-p_{h}^{n}, R_{h} u^{n}-u^{n}\right)+b\left(p^{n}-P_{h} p^{n}, u^{n}-u_{h}^{n}\right) \\
& +b\left(P_{h} p^{n}-p_{h}^{n}, u^{n}-u_{h}^{n}\right) .
\end{array}
$$

Since $P_{h} p^{n}-p_{h}^{n} \in M_{h}$, we have the following equality:

$$
b\left(P_{h} p^{n}-p_{h}^{n}, u^{n}-u_{h}^{n}\right)=0 .
$$

We have also:

$$
d\left(u^{n}-u_{h}^{n}, u^{n}-u_{h}^{n}\right)+d\left(u_{h}^{n}, \xi^{n}\right)-d\left(u^{n}, \xi^{n}\right)=d\left(u^{n}-u_{h}^{n}, u^{n}-u_{h}^{n}-\left(R_{h} u^{n}-u_{h}^{n}\right)\right),
$$

then

$$
d\left(u^{n}-u_{h}^{n}, u^{n}-u_{h}^{n}\right)+d\left(u_{h}^{n}, \xi^{n}\right)-d\left(u^{n}, \xi^{n}\right)=d\left(u^{n}-u_{h}^{n}, u^{n}-R_{h} u^{n}\right) .
$$

Finally, we have the following identity:

$$
\begin{array}{r}
\left(\bar{\partial}_{t} \xi^{n}, \xi^{n}\right)+\nu\left\|\nabla\left(u^{n}-u_{h}^{n}\right)\right\|_{0, \Omega}^{2}=\quad \frac{1}{2 k}\left(\left\|\xi^{n}\right\|_{0, \Omega}^{2}-\left\|\xi^{n-1}\right\|_{0, \Omega}^{2}+\left\|\xi^{n}-\xi^{n-1}\right\|_{0, \Omega}^{2}\right) \\
+\nu\left\|\nabla\left(u^{n}-u_{h}^{n}\right)\right\|_{0, \Omega}^{2}
\end{array}
$$

By using the previous lemmas, we have for all $\theta_{1}, \theta_{1}>0$ independents of $h$ and $k$ :

$$
\begin{aligned}
\left(\bar{\partial}_{t} \xi^{n}, \xi^{n}\right)+\nu\left\|\nabla\left(u^{n}-u_{h}^{n}\right)\right\|_{0, \Omega}^{2} \lesssim & \left(\left\|\nabla\left(u^{n}-R_{h} u^{n}\right)\right\|_{0, \Omega}^{2}+\left\|\bar{\partial}_{t} R_{h} u^{n}-\partial_{t} u^{n}\right\|_{0, \Omega}^{2}+\left\|p^{n}-P_{h} p^{n}\right\|_{1, \Omega}^{2}\right) \\
& +\left\|\xi^{n}\right\|_{0, \Omega}^{2}+\theta_{1}\left\|p^{n}-p_{h}^{n}\right\|_{1, \Omega}^{2}+\frac{\alpha^{2}}{2}\left\|\nabla\left(T^{n}-T_{h}^{n}\right)\right\|_{0, \Omega}^{2} \\
& +\left|a_{1}\left(u_{h}^{n-1}, u_{h}^{n}, \xi^{n}\right)-a_{1}\left(u^{n}, u^{n}, \xi^{n}\right)\right|+\theta_{2}\left\|\nabla\left(u^{n}-u_{h}^{n}\right)\right\|_{0, \Omega}^{2} .
\end{aligned}
$$


By using the previous lemmas, we obtain:

$$
\begin{aligned}
\frac{1}{2 k}\left(\left\|\xi^{n}\right\|_{0, \Omega}^{2}\right. & \left.-\left\|\xi^{n-1}\right\|_{0, \Omega}^{2}+\left\|\xi^{n}-\xi^{n-1}\right\|_{0, \Omega}^{2}\right)+\nu\left\|\nabla\left(u^{n}-u_{h}^{n}\right)\right\|_{0, \Omega}^{2} \\
& \lesssim\left(h^{2 \sigma}+k^{2}\right)+\left\|\nabla\left(T^{n}-T_{h}^{n}\right)\right\|_{0, \Omega}^{2}+\left\|\xi^{n}\right\|_{0, \Omega}^{2} \\
& +\left(N A+\theta_{0} N M+\theta_{2}+\frac{4 \theta_{1}}{\beta^{2}}(1+\rho)^{2}(\nu+N M)^{2}\right)\left\|\nabla\left(u^{n}-u_{h}^{n}\right)\right\|_{0, \Omega}^{2} \\
& +\left(N A+\frac{4 \theta_{1}}{\beta^{2}}(1+\rho)^{2} N^{2} A^{2}\right)\left\|\nabla\left(u^{n-1}-u_{h}^{n-1}\right)\right\|_{0, \Omega}^{2} \\
\frac{1}{2 k}\left(\left\|\xi^{n}\right\|_{0, \Omega}^{2}\right. & \left.-\left\|\xi^{n-1}\right\|_{0, \Omega}^{2}+\left\|\xi^{n}-\xi^{n-1}\right\|_{0, \Omega}^{2}\right)+\nu\left\|\nabla\left(u^{n}-u_{h}^{n}\right)\right\|_{0, \Omega}^{2} \\
& \lesssim\left(h^{2 \sigma}+k^{2}\right)+\left\|\nabla\left(T^{n}-T_{h}^{n}\right)\right\|_{0, \Omega}^{2}+\left\|\xi^{n}\right\|_{0, \Omega}^{2} \\
& +\left(N A+\theta_{0} N M+\theta_{2}+\frac{4 \theta_{1}}{\beta^{2}}(1+\rho)^{2}(\nu+N M)^{2}\right)\left\|\nabla\left(u^{n}-u_{h}^{n}\right)\right\|_{0, \Omega}^{2} \\
& +\left(N A+\frac{4 \theta_{1}}{\beta^{2}}(1+\rho)^{2} N^{2} A^{2}\right)\left\|\nabla\left(u^{n-1}-u_{h}^{n-1}\right)\right\|_{0, \Omega}^{2}
\end{aligned}
$$

Using the hypothesis, $N A<\nu+M N$, then for

$$
\theta_{0}=\theta_{2}=\frac{\nu}{8(1+N M)}
$$

and

$$
\theta_{1}=\nu \beta^{2}\left(32\left(1+\rho^{2}\right)(\nu+N M+N A)(\nu+N M-N A)\right)^{-1},
$$

we have:

$$
N A+\frac{4 \theta_{1}}{\beta^{2}}(1+\rho)^{2} N^{2} A^{2} \leq \frac{\nu}{4}
$$

and

$$
N A+\theta_{0} N M+\theta_{2}+\frac{4 \theta_{1}}{\beta^{2}}(1+\rho)^{2}(\nu+N M)^{2} \leq \frac{\nu}{2} .
$$

Therefore, we have:

$$
\begin{aligned}
\left\|\xi^{n}\right\|_{0, \Omega}^{2}+\nu k\left\|\nabla\left(u^{n}-u_{h}^{n}\right)\right\|_{0, \Omega}^{2} \lesssim & \left\|\xi^{n-1}\right\|_{0, \Omega}^{2}+2 k\left\|\xi^{n}\right\|_{0, \Omega}^{2}+k\left\|\nabla\left(T^{n}-T_{h}^{n}\right)\right\|_{0, \Omega}^{2} \\
& +\frac{\nu k}{2}\left\|\nabla\left(u^{n-1}-u_{h}^{n-1}\right)\right\|_{0, \Omega}^{2}+k\left(h^{2 \sigma}+k^{2}\right) .
\end{aligned}
$$

By summing over the time, we have:

$$
\begin{aligned}
\left\|\xi^{n}\right\|_{0, \Omega}^{2}+\nu k \sum_{i=1}^{n}\left\|\nabla\left(u^{i}-u_{h}^{i}\right)\right\|_{0, \Omega}^{2} \lesssim & 2 k \sum_{i=1}^{n}\left\|\xi^{n}\right\|_{0, \Omega}^{2}+k \sum_{i=1}^{n}\left\|\nabla\left(T^{i}-T_{h}^{i}\right)\right\|_{0, \Omega}^{2} \\
& +\frac{\nu k}{2} \sum_{i=1}^{n-1}\left\|\nabla\left(u^{i}-u_{h}^{i}\right)\right\|_{0, \Omega}^{2}+k n\left(h^{2 \sigma}+k^{2}\right) .
\end{aligned}
$$

If $2 k \leq \frac{1}{2}$, we obtain:

$$
\begin{aligned}
\left\|\xi^{n}\right\|_{0, \Omega}^{2}+2 \nu k\left\|\nabla\left(u^{n}-u_{h}^{n}\right)\right\|_{0, \Omega}^{2} \lesssim & k n\left(h^{2 \sigma}+k^{2}\right) \\
& +4 k \sum_{i=1}^{n-1}\left\|\xi^{i}\right\|_{0, \Omega}^{2}+k \sum_{i=1}^{n}\left\|\nabla\left(T^{i}-T_{h}^{i}\right)\right\|_{0, \Omega}^{2}
\end{aligned}
$$

Finally, by using Gronwal lemma with:

$$
\begin{gathered}
a_{n}=\left\|\xi^{n}\right\|_{0, \Omega}^{2}, \quad b_{n}=2 \nu k\left\|\nabla\left(u^{n}-u_{h}^{n}\right)\right\|_{0, \Omega}^{2}, \\
c_{n}=k n\left(h^{2 \sigma}+k^{2}\right)+k \sum_{i=1}^{n}\left\|\nabla\left(T^{i}-T_{h}^{i}\right)\right\|_{0, \Omega}^{2},
\end{gathered}
$$


we obtain:

$$
\left\|\xi^{n}\right\|_{0, \Omega}^{2}+2 \nu k\left\|\nabla\left(u^{n}-u_{h}^{n}\right)\right\|_{0, \Omega}^{2} \lesssim\left(k n\left(h^{2 \sigma}+k^{2}\right)+k \sum_{i=1}^{n}\left\|\nabla\left(T^{i}-T_{h}^{i}\right)\right\|_{0, \Omega}^{2}\right) \exp (4 k n)
$$

that we can rewrite it in the form:

$$
\begin{aligned}
\left\|u^{n}-u_{h}^{n}\right\|_{0, \Omega}^{2}+k\left\|\nabla\left(u^{n}-u_{h}^{n}\right)\right\|_{0, \Omega}^{2} & \leq\left\|u^{n}-R_{h} u^{n}\right\|_{0, \Omega}^{2}+\left\|\xi^{n}\right\|_{0, \Omega}^{2}+k\left\|\nabla\left(u^{n}-u_{h}^{n}\right)\right\|_{0, \Omega}^{2} \\
& \lesssim k n\left(h^{2 \sigma}+k^{2}\right)+k \sum_{i=1}^{n}\left\|\nabla\left(T^{i}-T_{h}^{i}\right)\right\|_{0, \Omega}^{2} .
\end{aligned}
$$

Now for temperature estimation we need the following lemmas.

Lemma 5.8. For all constants $\theta_{3}, \theta_{4}$ independents of $k$ and $h$, we have:

$$
\begin{aligned}
\left|a_{1}\left(u_{h}^{n-1}, T_{h}^{n}, \eta^{n}\right)-a_{1}\left(u^{n}, T^{n}, \eta^{n}\right)\right| \lesssim & \left(h^{2 \sigma}+k^{2}\right)+\left(\theta_{3} N M+N B\right)\left\|\nabla\left(T^{n}-T_{h}^{n}\right)\right\|_{0, \Omega}^{2} \\
& +\left(\theta_{4} N B+N B / 2\right)\left\|u^{n-1}-u_{h}^{n-1}\right\|_{0, \Omega}^{2} .
\end{aligned}
$$

Proof. We set $\eta^{n}=r_{h} T^{n}-T_{h}^{n}$. By the triangular inequality, we have:

$$
\begin{aligned}
& \left|a_{1}\left(u_{h}^{n-1}, T_{h}^{n}, \eta^{n}\right)-a_{1}\left(u^{n}, T^{n}, \eta^{n}\right)\right| \leq\left|a_{1}\left(u_{h}^{n-1}, T_{h}^{n}, \eta^{n}\right)-a_{1}\left(u_{h}^{n-1}, T^{n}, \eta^{n}\right)\right| \\
& \quad+\left|a_{1}\left(u_{h}^{n-1}, T^{n}, \eta^{n}\right)-a_{1}\left(u^{n-1}, T^{n}, \eta^{n}\right)\right|+\left|a_{1}\left(u^{n-1}, T^{n}, \eta^{n}\right)-a_{1}\left(u^{n}, T^{n}, \eta^{n}\right)\right| \\
& \leq N M\left\|\nabla\left(T^{n}-T_{h}^{n}\right)\right\|_{0, \Omega}\left\|\nabla\left(T_{h}^{n}-r_{h} T^{n}\right)\right\|_{0, \Omega}+N B\left\|\nabla\left(u^{n-1}-u_{h}^{n-1}\right)\right\|_{0, \Omega}\left\|\nabla\left(T^{n}-r_{h} T^{n}\right)\right\|_{0, \Omega} \\
& +N B\left\|\nabla\left(u^{n-1}-u_{h}^{n-1}\right)\right\|_{0, \Omega}\left\|\nabla\left(T^{n}-T_{h}^{n}\right)\right\|_{0, \Omega}+N B\left\|\nabla\left(u^{n}-u^{n-1}\right)\right\|_{0, \Omega}\left\|\nabla\left(T^{n}-r_{h} T^{n}\right)\right\|_{0, \Omega} \\
& +N B\left\|\nabla\left(u^{n}-u^{n-1}\right)\right\|_{0, \Omega}\left\|\nabla\left(T^{n}-T_{h}^{n}\right)\right\|_{0, \Omega},
\end{aligned}
$$

then

$$
\begin{gathered}
\left|a_{1}\left(u_{h}^{n-1}, T_{h}^{n}, \eta^{n}\right)-a_{1}\left(u^{n}, T^{n}, \eta^{n}\right)\right| \leq\left(\theta_{3} N M+N B\right)\left\|\nabla\left(T^{n}-T_{h}^{n}\right)\right\|_{0, \Omega}^{2}+\left\|\nabla\left(T^{n}-r_{h} T^{n}\right)\right\|_{0, \Omega}^{2} \\
\left(\theta_{4} N B+N B / 2\right)\left\|\nabla\left(u_{h}^{n-1}-u^{n-1}\right)\right\|_{0, \Omega}^{2}+\left\|\nabla\left(u^{n}-u^{n-1}\right)\right\|_{0, \Omega}^{2} \\
\lesssim\left(h^{2 \sigma}+k^{2}\right)+\left(\theta_{3} N M+N B\right)\left\|\nabla\left(T^{n}-T_{h}^{n}\right)\right\|_{0, \Omega}^{2}+\left(\theta_{4} N B+N B / 2\right)\left\|u^{n-1}-u_{h}^{n-1}\right\|_{0, \Omega}^{2} .
\end{gathered}
$$

Lemma 5.9. We have the following a priori estimate:

$$
\left\|\bar{\partial}_{t} r_{h} T^{n}-\partial_{t} T^{n}\right\|_{0} \lesssim\left(h^{\sigma}+k\right) .
$$

Proof. By applying the development of Taylor with remainder integral, we obtain:

$$
\begin{aligned}
\left\|\bar{\partial}_{t} r_{h} T^{n}-\partial_{t} T^{n}\right\|_{0, \Omega} \leq & \left\|\bar{\partial}_{t} r_{h} T^{n}-\bar{\partial}_{t} T^{n}\right\|_{0, \Omega}+\left\|\bar{\partial}_{t} T^{n}-\partial_{t} T^{n}\right\|_{0, \Omega} \\
= & \left\|\frac{1}{k} \int_{t_{n-1}}^{t_{n}}\left(r_{h} \partial_{t} T(s)-\partial_{t} T(s)\right) d s\right\|_{0, \Omega} \\
& +\left\|\int_{t_{n-1}}^{t_{n}}\left(s-t^{n-1}\right) \partial_{t^{2}}^{2} T(s) d s\right\|_{0, \Omega} \\
\lesssim & \left(h^{\sigma}\|T\|_{W^{1, \infty}\left(H^{\sigma}\right)}+k\|T\|_{W^{2, \infty}\left(L^{2}\right)}\right) \\
& \lesssim\left(h^{\sigma}+k\right) .
\end{aligned}
$$


Lemma 5.10. For all constants $\theta_{3}, \theta_{4}, \theta_{5}, \theta_{6}, \theta_{7}$, independents of $h$ and $k$, we have:

$$
\begin{aligned}
\frac{1}{2 k}\left(\left\|\eta^{n}\right\|_{0, \Omega}^{2}-\right. & \left.\left\|\eta^{n-1}\right\|_{0, \Omega}^{2}+\left\|\eta^{n}-\eta^{n-1}\right\|_{0, \Omega}^{2}\right)+\lambda\left\|\nabla\left(T^{n}-T_{h}^{n}\right)\right\|_{0, \Omega}^{2} \lesssim\left(h^{2 \sigma}+k^{2}\right) \\
& +\left(\theta_{5}+\theta_{6}+\theta_{7}+\theta_{8}\right)\left\|\eta^{n}\right\|_{0, \Omega}^{2}+\left(\theta_{3} N M+N B\right)\left\|\nabla\left(T^{n}-T_{h}^{n}\right)\right\|_{0, \Omega}^{2} \\
& +\rho^{2} \frac{M_{C}^{2} C_{g}^{2}}{\theta_{8}}\left\|\nabla\left(T^{n-1}-T_{h}^{n-1}\right)\right\|_{0, \Omega}^{2}+\frac{\rho^{2}}{4 \theta_{6}}\left\|\nabla\left(C_{1}^{n}-C_{1_{h}}^{n}\right)\right\|_{0, \Omega}^{2} \\
& +\frac{\rho^{2}}{4 \theta_{7}}\left\|\nabla\left(C_{2}^{n}-C_{2_{h}}^{n}\right)\right\|_{0, \Omega}^{2}+\left(\theta_{4} N B+\frac{N B}{2}\right)\left\|\nabla\left(u^{n-1}-u_{h}^{n-1}\right)\right\|_{0, \Omega}^{2} .
\end{aligned}
$$

Proof. We set $\eta^{n}=r_{h} T^{n}-T_{h}^{n}$. First of all, we have the two following equalities:

$$
\left(\bar{\partial}_{t} \eta^{n}, \eta^{n}\right)+\lambda d\left(T^{n}-T_{h}^{n}, T^{n}-T_{h}^{n}\right)=\left(\bar{\partial}_{t} r_{h} T^{n}-\bar{\partial}_{t} T_{h}^{n}, \eta^{n}\right)+\lambda d\left(T^{n}-T_{h}^{n}, T^{n}-T_{h}^{n}\right),
$$

and

$$
\left(\bar{\partial}_{t} T_{h}^{n}, \eta^{n}\right)=-\lambda d\left(T_{h}^{n}, \eta^{n}\right)-a_{1}\left(u_{h}^{n-1}, T_{h}^{n}, \eta^{n}\right)+K_{1}\left(C_{1_{h}}^{n}, T_{h}^{n-1}, \eta^{n}\right)+K_{2}\left(C_{2_{h}}^{n}, T_{h}^{n-1}, \eta^{n}\right) .
$$

Therefore, we have:

$$
\begin{aligned}
\left(\bar{\partial}_{t} \eta^{n}, \eta^{n}\right)+\lambda d\left(T^{n}-T_{h}^{n}, T^{n}-T_{h}^{n}\right)=\left(\bar{\partial}_{t} r_{h} T^{n}, \eta^{n}\right)+\lambda d\left(T_{h}^{n}, \eta^{n}\right)+\lambda d\left(T^{n}-T_{h}^{n}, T^{n}-T_{h}^{n}\right) & \\
& +a_{1}\left(u_{h}^{n-1}, T_{h}^{n}, \eta^{n}\right)-K_{1}\left(C_{1_{h}}^{n}, T_{h}^{n-1}, \eta^{n}\right)-K\left(C_{2_{h}}^{n}, T_{h}^{n-1}, \eta^{n}\right)
\end{aligned}
$$

then:

$$
\begin{aligned}
\left(\bar{\partial}_{t} \eta^{n}, \eta^{n}\right)+ & \lambda d\left(T^{n}-T_{h}^{n}, T^{n}-T_{h}^{n}\right)=\left(\bar{\partial}_{t} r_{h} T^{n}-\partial_{t} T^{n}, \eta^{n}\right)+\lambda d\left(T_{h}^{n}-T^{n}, \eta^{n}\right) \\
& +\lambda d\left(T^{n}-T_{h}^{n}, T^{n}-T_{h}^{n}\right)+a_{1}\left(u_{h}^{n-1}, T_{h}^{n}, \eta^{n}\right)-a_{1}\left(u^{n}, T^{n}, \eta^{n}\right) \\
& +K_{1}\left(C_{1}^{n}, T^{n}, \eta^{n}\right)-K\left(C_{1_{h}}^{n}, T_{h}^{n-1}, \eta^{n}\right)+K_{2}\left(C_{2}^{n}, T^{n}, \eta^{n}\right)-K_{2}\left(C_{2_{h}}^{n}, T_{h}^{n-1}, \eta^{n}\right) .
\end{aligned}
$$

However:

$$
\left\{\begin{array}{r}
d\left(T^{n}-T_{h}^{n}, T^{n}-T_{h}^{n}\right)+d\left(T_{h}^{n}-T^{n}, \eta^{n}\right)=d\left(T^{n}-T_{h}^{n}, T^{n}-r_{h} T^{n}\right) \\
=d\left(T^{n}-r_{h} T^{n}, T^{n}-r_{h} T^{n}\right)+d\left(r_{h} T^{n}-T_{h}^{n}, T^{n}-r_{h} T^{n}\right)
\end{array}\right.
$$

by using the identity:

$$
a(a-b)=\frac{1}{2}\left(a^{2}-b^{2}+(a-b)^{2}\right)
$$

we have:

$$
\begin{aligned}
\frac{1}{2 k}\left(\left\|\eta^{n}\right\|_{0, \Omega}^{2}\right. & \left.-\left\|\eta^{n-1}\right\|_{0, \Omega}^{2}+\left\|\eta^{n}-\eta^{n-1}\right\|_{0, \Omega}^{2}\right)+\lambda\left\|\nabla\left(T^{n}-T_{h}^{n}\right)\right\|_{0, \Omega}^{2} \\
& =\left(\bar{\partial}_{t} r_{h} T^{n}-\partial_{t} u^{n}, \eta^{n}\right)+\lambda\left\|\nabla\left(T^{n}-r_{h} T^{n}\right)\right\|_{0, \Omega}^{2} \\
& +a_{1}\left(u_{h}^{n-1}, T_{h}^{n}, \eta^{n}\right)-a_{1}\left(u^{n}, T^{n}, \eta^{n}\right) \\
& +K_{1}\left(C_{1}^{n}, T^{n}, \eta^{n}\right)-K_{1}\left(C_{1_{h}}^{n}, T_{h}^{n-1}, \eta^{n}\right) \\
& +K_{2}\left(C_{2}^{n}, T^{n}, \eta^{n}\right)-K_{2}\left(C_{2_{h}}^{n}, T_{h}^{n-1}, \eta^{n}\right)
\end{aligned}
$$


We have also:

$$
\begin{aligned}
K_{1}\left(C_{1}^{n}, T^{n}, \eta^{n}\right)-K_{1}\left(C_{1_{h}}^{n}, T_{h}^{n-1}, \eta^{n}\right)= & K\left(C_{1}^{n}, T^{n}, \eta^{n}\right)-K_{1}\left(C_{1}^{n}, T^{n-1}, \eta^{n}\right) \\
& +K_{1}\left(C_{1}^{n}, T^{n-1}, \eta^{n}\right)-K_{1}\left(C_{1_{h}}^{n}, T^{n-1}, \eta^{n}\right) \\
& +K_{1}\left(C_{1_{h}}^{n}, T^{n-1}, \eta^{n}\right)-K_{1}\left(C_{1_{h}}^{n}, T_{h}^{n-1}, \eta^{n}\right) \\
\lesssim & k\|T\|_{W^{1, \infty}}\left\|\eta^{n}\right\|_{0, \Omega}+\rho\left\|\nabla\left(C_{1}^{n}-C_{1_{h}}^{n}\right)\right\|_{0, \Omega}\left\|\eta^{n}\right\|_{0, \Omega} \\
& +\rho C_{g} M_{C}\left\|\nabla\left(T^{n-1}-T_{h}^{n-1}\right)\right\|_{0, \Omega}\left\|\eta^{n}\right\|_{0, \Omega},
\end{aligned}
$$

we have same inequality for the second concentration. We have also:

$$
\begin{aligned}
\frac{1}{2 k}\left(\left\|\eta^{n}\right\|_{0, \Omega}^{2}\right. & \left.-\left\|\eta^{n-1}\right\|_{0, \Omega}^{2}+\left\|\eta^{n}-\eta^{n-1}\right\|_{0, \Omega}^{2}\right)+\lambda\left\|\nabla\left(T^{n}-T_{h}^{n}\right)\right\|_{0, \Omega}^{2} \\
& \lesssim\left(h^{\sigma}+k\right)\left\|\eta^{n}\right\|_{0, \Omega}+\lambda\left\|\nabla\left(T^{n}-r_{h} T^{n}\right)\right\|_{0, \Omega}^{2}+ \\
& \left(h^{2 \sigma}+k^{2}\right)+\left(\theta_{3} N M+N B\right)\left\|\nabla\left(T^{n}-T_{h}^{n}\right)\right\|_{0, \Omega}^{2} \\
& +\left(\theta_{4} N B+\frac{N B}{2}\right)\left\|\nabla\left(u^{n-1}-u_{h}^{n-1}\right)\right\|_{0, \Omega}^{2} \\
& +2 k\left\|\eta^{n}\right\|_{0, \Omega}+\rho\left\|\nabla\left(C_{1}^{n}-C_{1_{h}}^{n}\right)\right\|_{0, \Omega}\left\|\eta^{n}\right\|_{0, \Omega}+\rho\left\|\nabla\left(C_{2}^{n}-C_{2_{h}}^{n}\right)\right\|_{0, \Omega}\left\|\eta^{n}\right\|_{0, \Omega} \\
& +2 \rho M_{C} C_{g}\left\|\nabla\left(T^{n-1}-T_{h}^{n-1}\right)\right\|_{0, \Omega}\left\|\eta^{n}\right\|_{0, \Omega} .
\end{aligned}
$$

Therefore:

$$
\begin{aligned}
\frac{1}{2 k}\left(\left\|\eta^{n}\right\|_{0, \Omega}^{2}\right. & \left.-\left\|\eta^{n-1}\right\|_{0, \Omega}^{2}+\left\|\eta^{n}-\eta^{n-1}\right\|_{0, \Omega}^{2}\right)+\lambda\left\|\nabla\left(T^{n}-T_{h}^{n}\right)\right\|_{0, \Omega}^{2} \\
& \lesssim\left(h^{2 \sigma}+k^{2}\right)+\left(\theta_{5}+\theta_{6}+\theta_{7}+\theta_{8}\right)\left\|\eta^{n}\right\|_{0, \Omega}^{2}+\left(\theta_{3} N M+N B\right)\left\|\nabla\left(T^{n}-T_{h}^{n}\right)\right\|_{0, \Omega}^{2} \\
& +\rho^{2} \frac{M_{C}^{2} C_{g}^{2}}{\theta_{8}}\left\|\nabla\left(T^{n-1}-T_{h}^{n-1}\right)\right\|_{0, \Omega}^{2}+\frac{\rho^{2}}{4 \theta_{6}}\left\|\nabla\left(C_{1}^{n}-C_{1_{h}}^{n}\right)\right\|_{0, \Omega}^{2}+\frac{\rho^{2}}{4 \theta_{7}}\left\|\nabla\left(C_{2}^{n}-C_{2_{h}}^{n}\right)\right\|_{0, \Omega}^{2} \\
& +\left(\theta_{4} N B+\frac{N B}{2}\right)\left\|\nabla\left(u^{n-1}-u_{h}^{n-1}\right)\right\|_{0, \Omega}^{2} .
\end{aligned}
$$

Lemma 5.11. We assume that $N B<\frac{\lambda}{2}, 6 \rho^{2} M_{C}^{2} C_{g}^{2} \leq \lambda$ and $\left(k \leq \frac{1}{4}\right)$. Then we have:

$$
\begin{aligned}
\left\|T^{n}-T_{h}^{n}\right\|_{0, \Omega}^{2} & +\lambda k\left\|\nabla\left(T^{n}-T_{h}^{n}\right)\right\|_{0, \Omega}^{2} \lesssim k n\left(h^{2 \sigma}+k^{2}\right)+2 k\left(\theta_{4} N B+\frac{N B}{2}\right) \sum_{i=1}^{n-1}\left\|\nabla\left(u^{i}-u_{h}^{i}\right)\right\|_{0, \Omega}^{2} \\
& +8 \rho^{2} k \sum_{i=1}^{n}\left\|\nabla\left(C_{1}^{i}-C_{1_{h}}^{i}\right)\right\|_{0, \Omega}^{2}+8 \rho^{2} k \sum_{i=1}^{n}\left\|\nabla\left(C_{2}^{i}-C_{2_{h}}^{i}\right)\right\|_{0, \Omega}^{2} .
\end{aligned}
$$

Proof. By choosing $\theta_{5}=\theta_{6}=\theta_{7}=\theta_{8}=\frac{1}{16}$ and $\theta_{3}=\frac{\lambda-2 N B}{2 N M}$, we have:

$$
\begin{aligned}
\frac{1}{2 k}\left(\left\|\eta^{n}\right\|_{0, \Omega}^{2}\right. & \left.-\left\|\eta^{n-1}\right\|_{0, \Omega}^{2}+\left\|\eta^{n}-\eta^{n-1}\right\|_{0, \Omega}^{2}\right)+\frac{\lambda}{2}\left\|\nabla\left(T^{n}-T_{h}^{n}\right)\right\|_{0, \Omega}^{2} \lesssim \frac{1}{4}\left\|\eta^{n}\right\|_{0, \Omega}^{2} \\
& +\left(h^{2 \sigma}+k^{2}\right)+16 \rho^{2} M_{C}^{2} C_{g}^{2}\left\|\nabla\left(T^{n-1}-T_{h}^{n-1}\right)\right\|_{0, \Omega}^{2}+4 \rho^{2}\left\|\nabla\left(C_{1}^{n}-C_{1_{h}}^{n}\right)\right\|_{0, \Omega}^{2} \\
& +4 \rho^{2}\left\|\nabla\left(C_{2}^{n}-C_{2_{h}}^{n}\right)\right\|_{0, \Omega}^{2}+\left(\theta_{4} N B+\frac{N B}{2}\right)\left\|\nabla\left(u^{n-1}-u_{h}^{n-1}\right)\right\|_{0, \Omega}^{2},
\end{aligned}
$$


by summing over the time, we have:

$$
\begin{aligned}
\frac{1}{2 k}\left(\left\|\eta^{n}\right\|_{0, \Omega}^{2}\right) & +\frac{\lambda}{2} \sum_{i=1}^{n}\left\|\nabla\left(T^{i}-T_{h}^{i}\right)\right\|_{0, \Omega}^{2} \lesssim \frac{1}{4} \sum_{i=1}^{n}\left\|\eta^{i}\right\|_{0, \Omega}^{2}+n\left(h^{2 \sigma}+k^{2}\right) \\
& +16 \rho^{2} M_{C}^{2} C_{g}^{2} \sum_{i=1}^{n-1}\left\|\nabla\left(T^{i}-T_{h}^{i}\right)\right\|_{0, \Omega}^{2}+4 \rho^{2} \sum_{i=1}^{n}\left\|\nabla\left(C_{1}^{i}-C_{1_{h}}^{i}\right)\right\|_{0, \Omega}^{2} \\
& +4 \rho^{2} \sum_{i=1}^{n}\left\|\nabla\left(C_{2}^{i}-C_{2_{h}}^{i}\right)\right\|_{0, \Omega}^{2}+\left(\theta_{4} N B+\frac{N B}{2}\right) \sum_{i=1}^{n-1}\left\|\nabla\left(u^{i}-u_{h}^{i}\right)\right\|_{0, \Omega}^{2} .
\end{aligned}
$$

However:

$$
16 \rho^{2} M_{C}^{2} C_{g}^{2} \leq \frac{\lambda}{2} \text { and } k \leq \frac{1}{4}
$$

We deduce that:

$$
\begin{aligned}
\frac{7}{8}\left(\left\|\eta^{n}\right\|_{0, \Omega}^{2}\right) & +\lambda k\left\|\nabla\left(T^{n}-T_{h}^{n}\right)\right\|_{0, \Omega}^{2} \lesssim \frac{k}{2} \sum_{i=1}^{n-1}\left\|\eta^{i}\right\|_{0, \Omega}^{2}+k n\left(h^{2 \sigma}+k^{2}\right)+8 \rho^{2} k \sum_{i=1}^{n}\left\|\nabla\left(C_{1}^{i}-C_{1_{h}}^{i}\right)\right\|_{0, \Omega}^{2} \\
& +8 \rho^{2} k \sum_{i=1}^{n}\left\|\nabla\left(C_{2}^{i}-C_{2_{h}}^{i}\right)\right\|_{0, \Omega}^{2}+2 k\left(\theta_{4} N B+\frac{N B}{2}\right) \sum_{i=1}^{n-1}\left\|\nabla\left(u^{i}-u_{h}^{i}\right)\right\|_{0, \Omega}^{2} .
\end{aligned}
$$

By using the discret Gronwal lemma, we obtain:

$$
\begin{aligned}
\left\|\eta^{n}\right\|_{0, \Omega}^{2}+k \lambda\left\|\nabla\left(T^{n}-T_{h}^{n}\right)\right\|_{0, \Omega}^{2} \lesssim & \left(k n\left(h^{2 \sigma}+k^{2}\right)+2 k\left(\theta_{4} N B+\frac{N B}{2}\right) \sum_{i=1}^{n-1}\left\|\nabla\left(u^{i}-u_{h}^{i}\right)\right\|_{0, \Omega}^{2}\right. \\
& \left.+8 \rho^{2} k \sum_{i=1}^{n}\left\|\nabla\left(C_{1}^{i}-C_{1_{h}}^{i}\right)\right\|_{0, \Omega}^{2}+8 \rho^{2} k \sum_{i=1}^{n}\left\|\nabla\left(C_{2}^{i}-C_{2_{h}}^{i}\right)\right\|_{0, \Omega}^{2}\right) \\
& \times \exp (k n) .
\end{aligned}
$$

The result comes from the triangular inequality:

$$
\begin{aligned}
\left\|T^{n}-T_{h}^{n}\right\|_{0, \Omega}^{2}+k \lambda\left\|\nabla\left(T^{n}-T_{h}^{n}\right)\right\|_{0, \Omega}^{2} & \leq\left\|T^{n}-r_{h} T^{n}\right\|_{0, \Omega}^{2}+\left\|\eta^{n}\right\|_{0, \Omega}^{2}+k \lambda\left\|\nabla\left(T^{n}-T_{h}^{n}\right)\right\|_{0, \Omega}^{2} \\
& \lesssim h^{\sigma}\|T\|_{L^{\infty}\left(0, t, H^{\sigma}\right)}+\left\|\eta^{n}\right\|_{0, \Omega}^{2}+k \lambda\left\|\nabla\left(T^{n}-T_{h}^{n}\right)\right\|_{0, \Omega}^{2} .
\end{aligned}
$$

Lemma 5.12. For all constants $\theta_{8}, \theta_{9}, \theta_{11}$ and $\theta_{12}$ independents of $h$ and $k$, we have:

$$
\begin{aligned}
& \left\{\begin{array}{r}
\left|a_{1}\left(u_{h}^{n-1}, C_{1_{h}}^{n}, \epsilon_{1}^{n}\right)-a_{1}\left(u^{n}, C_{1}^{n}, \epsilon_{1}^{n}\right)\right| \lesssim\left(\begin{array}{r}
2 \sigma \\
2 \sigma
\end{array}\right)+\left(\theta_{9} N M+N M_{C}\right)\left\|\nabla\left(C_{1}^{n}-C_{1_{h}}^{n}\right)\right\|_{0, \Omega}^{2} \\
+\left(\theta_{10} N M_{C}+N M_{C} / 2\right)\left\|\nabla\left(u^{n-1}-u_{h}^{n-1}\right)\right\|_{0, \Omega}^{2} .
\end{array}\right.
\end{aligned}
$$

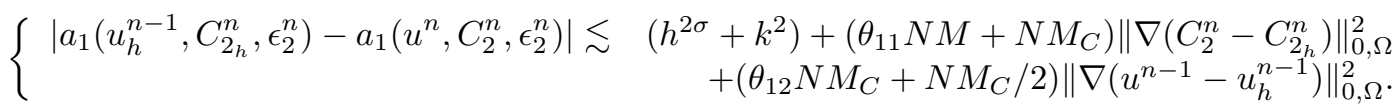

Proof. We set $\epsilon_{1}^{n}=r_{h} C_{1}^{n}-C_{1_{h}}^{n}$. By using the previous lemmas and the Young inequalities, we have:

$$
\begin{aligned}
& \left|a_{1}\left(u_{h}^{n-1}, C_{1_{h}}^{n}, \epsilon_{1}^{n}\right)-a_{1}\left(u^{n}, C_{1}^{n}, \epsilon_{1}^{n}\right)\right| \leq\left|a_{1}\left(u_{h}^{n-1}, C_{1_{h}}^{n}, \epsilon_{1}^{n}\right)-a_{1}\left(u_{h}^{n-1}, C_{1}^{n}, \epsilon_{1}^{n}\right)\right| \\
& \quad+\left|a_{1}\left(u_{h}^{n-1}, C_{1}^{n}, \epsilon_{1}^{n}\right)-a_{1}\left(u^{n-1}, C_{1}^{n}, \epsilon_{1}^{n}\right)\right|+\left|a_{1}\left(u^{n-1}, C_{1}^{n}, \epsilon_{1}^{n}\right)-a_{1}\left(u^{n}, C_{1}^{n}, \epsilon_{1}^{n}\right)\right| \\
& \quad \leq N\left\|\nabla\left(C_{1}^{n}-C_{1_{h}}^{n}\right)\right\|_{0, \Omega}\left\|\nabla\left(C_{1_{h}}^{n}-r_{h} C_{1}^{n}\right)\right\|_{0, \Omega}+N M_{C} \mid \nabla\left(u^{n-1}-u_{h}^{n-1}\right)\left\|_{0, \Omega}\right\| \nabla\left(C_{1}^{n}-r_{h} C_{1}^{n}\right) \|_{0, \Omega} \\
& \quad+N M_{C}\left\|\nabla\left(u^{n-1}-u_{h}^{n-1}\right)\right\|_{0, \Omega}\left\|\nabla\left(C_{1}^{n}-C_{1_{h}}^{n}\right)\right\|_{0, \Omega}+N M_{C}\left\|\nabla\left(u^{n}-u^{n-1}\right)\right\|_{0, \Omega}\left\|\nabla\left(C_{1}^{n}-r_{h} C_{1}^{n}\right)\right\|_{0, \Omega} \\
& \quad+N M_{C}\left\|\nabla\left(u^{n}-u^{n-1}\right)\right\|_{0, \Omega}\left\|\nabla\left(C_{1}^{n}-C_{1_{h}}^{n}\right)\right\|_{0, \Omega} \\
& \leq\left(\theta_{8} N M_{+}+N M_{C}\right)\left\|\nabla\left(C_{1}^{n}-C_{1_{h}}^{n}\right)\right\|_{0, \Omega}^{2}+C_{1}\left\|\nabla\left(C_{1}^{n}-r_{h} C_{1}^{n}\right)\right\|_{0, \Omega}^{2} \\
& \quad\left(\theta_{9} N M_{C}+N M_{C} / 2\right)\left\|\nabla\left(u_{h}^{n-1}-u^{n-1}\right)\right\|_{0, \Omega}^{2}+C_{1}\left\|\nabla\left(u^{n}-u^{n-1}\right)\right\|_{0, \Omega}^{2} \\
& \vdots\left(h^{2 \sigma}+k^{2}\right)+\left(\theta_{8} N M+N M_{C}\right)\left\|\nabla\left(C_{1}^{n}-C_{1_{h}}^{n}\right)\right\|_{0, \Omega}^{2}+\left(\theta_{9} N M_{C}+N M_{C} / 2\right)\left\|u^{n-1}-u_{h}^{n-1}\right\|_{0, \Omega}^{2} .
\end{aligned}
$$

The proof of the second inequality of the lemma is similar to the proof of the first one. 
We have the following lemma:

Lemma 5.13. We have the following estimate:

$$
\left\|\partial_{t} r_{h} C_{i}^{n}-\partial_{t} C_{i}^{n}\right\|_{0, \Omega} \lesssim\left(h^{\sigma}+k\right) . \quad(i=1,2)
$$

Proof. By applying the Taylor development with remainder integral, we obtain:

$$
\begin{aligned}
\left\|\bar{\partial}_{t} r_{h} C_{i}^{n}-\partial_{t} C_{i}^{n}\right\|_{0, \Omega} \leq & \left\|\bar{\partial}_{t} r_{h} C_{i}^{n}-\bar{\partial}_{t} C_{i}^{n}\right\|_{0, \Omega}+\left\|\bar{\partial}_{t} C_{i}^{n}-\partial_{t} C_{i}^{n}\right\|_{0, \Omega} \\
= & \left\|\frac{1}{k} \int_{t_{n-1}}^{t_{n}}\left(r_{h} \partial_{t} C_{i}(s)-\partial_{t} C_{i}(s)\right) d s\right\|_{0, \Omega} \\
& +\left\|\int_{t_{n-1}}^{t_{n}}\left(s-t^{n-1}\right) \partial_{t^{2}}^{2} C_{i}(s) d s\right\|_{0, \Omega} \\
\lesssim & \left(h^{\sigma}\left\|C_{i}\right\|_{W^{1, \infty}\left(H^{\sigma}\right)}+k\left\|C_{i}\right\|_{W^{2, \infty}\left(L^{2}\right)}\right) \\
\lesssim & \left(h^{\sigma}+k\right) .
\end{aligned}
$$

we have the same lemma for the second concentration.

Lemma 5.14. For all constants $\theta_{9}, \theta_{10}, \theta_{13}, \theta_{14}, \theta_{15}$, independents of $h$ and $k$, we have:

$$
\begin{aligned}
\frac{1}{2 k} & \left(\left\|\epsilon^{n}\right\|_{0, \Omega}^{2}-\left\|\epsilon^{n-1}\right\|_{0, \Omega}^{2}+\left\|\epsilon^{n}-\epsilon^{n-1}\right\|_{0, \Omega}^{2}\right)+\lambda\left\|\nabla\left(C_{1}^{n}-C_{1_{h}}^{n}\right)\right\|_{0, \Omega}^{2} \lesssim\left(h^{2 \sigma}+k^{2}\right) \\
& +\left(\theta_{13}+\theta_{14}+\theta_{15}\right)\left\|\epsilon^{n}\right\|_{0, \Omega}^{2}+\left(\theta_{9} N M+N M_{C}\right)\left\|\nabla\left(C_{1}^{n}-C_{1_{h}}^{n}\right)\right\|_{0, \Omega}^{2}+\rho^{2} \frac{M_{C}^{2} C_{g}^{2}}{4 \theta_{7}}\left\|\nabla\left(T^{n}-T_{h}^{n-1}\right)\right\|_{0, \Omega}^{2} \\
& +\frac{\rho^{2}}{4 \theta_{6}}\left\|\nabla\left(C_{1}^{n}-C_{1_{h}}^{n-1}\right)\right\|_{0, \Omega}^{2}\left(\theta_{10} N M_{C}+\frac{N M_{C}}{2}\right)\left\|\nabla\left(u^{n-1}-u_{h}^{n-1}\right)\right\|_{0, \Omega}^{2} .
\end{aligned}
$$

Proof. We set $\epsilon^{n}=r_{h} C_{1}^{n}-C_{1_{h}}^{n}$. First of all, we have:

$$
\left(\bar{\partial}_{t} \epsilon^{n}, \epsilon^{n}\right)+\lambda d\left(C_{1}^{n}-C_{1_{h}}^{n}, C_{1}^{n}-C_{1_{h}}^{n}\right)=\left(\bar{\partial}_{t} r_{h} C_{1}^{n}-\bar{\partial}_{t} C_{1_{h}}^{n}, \epsilon^{n}\right)+\lambda d\left(C_{1}^{n}-C_{1_{h}}^{n}, C_{1}^{n}-C_{1_{h}}^{n}\right)
$$

however

$$
\left(\bar{\partial}_{t} C_{1_{h}}^{n}, \epsilon^{n}\right)=-\lambda d\left(C_{1_{h}}^{n}, \epsilon^{n}\right)-a_{1}\left(u_{h}^{n-1}, C_{1_{h}}^{n}, \epsilon^{n}\right)-K\left(C_{1_{h}}^{n}, T_{h}^{n-1}, \epsilon^{n}\right) .
$$

Therefore:

$$
\begin{aligned}
\left(\bar{\partial}_{t} \epsilon^{n}, \epsilon^{n}\right)+\lambda d\left(C_{1}^{n}-\right. & \left.C_{1_{h}}^{n}, C_{1}^{n}-C_{1_{h}}^{n}\right)=\left(\bar{\partial}_{t} r_{h} C_{1}^{n}, \epsilon^{n}\right)+\lambda d\left(C_{1_{h}}^{n}, \epsilon^{n}\right)+\lambda d\left(C_{1}^{n}-C_{1_{h}}^{n}, C_{1}^{n}-C_{1_{h}}^{n}\right) \\
& +a_{1}\left(u_{h}^{n-1}, C_{1_{h}}^{n}, \epsilon^{n}\right)+K\left(C_{1_{h}}^{n}, T_{h}^{n-1}, \epsilon^{n}\right) \\
= & \left(\bar{\partial}_{t} r_{h} C_{1}^{n}-\partial_{t} C_{1}^{n}, \epsilon^{n}\right)+\lambda d\left(C_{1_{h}}^{n}-C_{1}^{n}, \epsilon^{n}\right)+\lambda d\left(C_{1}^{n}-C_{1_{h}}^{n}, C_{1}^{n}-C_{1_{h}}^{n}\right) \\
& +a_{1}\left(u_{h}^{n-1}, C_{1_{h}}^{n}, \epsilon^{n}\right)-a_{1}\left(u^{n}, C_{1}^{n}, \epsilon^{n}\right)-K\left(C_{1}^{n}, T^{n}, \epsilon^{n}\right)+K\left(C_{1_{h}}^{n}, T_{h}^{n-1}, \epsilon^{n}\right) .
\end{aligned}
$$

However:

$$
\begin{aligned}
d\left(C_{1}^{n}-C_{1_{h}}^{n}, C_{1}^{n}-C_{1_{h}}^{n}\right)+d\left(C_{1_{h}}^{n}-C_{1}^{n}, \epsilon^{n}\right)=d\left(C_{1}^{n}-C_{1_{h}}^{n}, C_{1}^{n}-r_{h} C_{1}^{n}\right) \\
=d\left(C_{1}^{n}-r_{h} C_{1}^{n}, C_{1}^{n}-r_{h} C_{1}^{n}\right)+d\left(r_{h} C_{1}^{n}-C_{1_{h}}^{n}, C_{1}^{n}-r_{h} C_{1}^{n}\right)
\end{aligned}
$$

and

$$
a(a-b)=\frac{1}{2}\left(a^{2}-b^{2}+(a-b)^{2}\right) .
$$

Therefore, we have:

$$
\begin{gathered}
\frac{1}{2 k}\left(\left\|\epsilon^{n}\right\|^{2}-\left\|\epsilon^{n-1}\right\|^{2}+\left\|\epsilon^{n}-\epsilon^{n-1}\right\|^{2}\right)+\lambda\left\|\nabla\left(C_{1}^{n}-C_{1_{h}}^{n}\right)\right\|^{2}=\left(\bar{\partial}_{t} r_{h} C_{1}^{n}-\partial_{t} u^{n}, \epsilon^{n}\right) \\
+\lambda\left\|\nabla\left(C_{1}^{n}-r_{h} C_{1}^{n}\right)\right\|^{2}+a_{1}\left(u_{h}^{n-1}, C_{1_{h}}^{n}, \epsilon^{n}\right)-a_{1}\left(u^{n}, C_{1}^{n}, \epsilon^{n}\right) \\
-K\left(C_{1}^{n}, T^{n}, \epsilon^{n}\right)+K\left(C_{1_{h}}^{n}, T_{h}^{n-1}, \epsilon^{n}\right) .
\end{gathered}
$$


We have as well:

$$
\begin{aligned}
K\left(C_{1}^{n}, T^{n}, \epsilon^{n}\right)-K\left(C_{1_{h}}^{n}, T_{h}^{n-1}, \epsilon^{n}\right)= & K\left(C_{1}^{n}, T^{n}, \epsilon^{n}\right)-K\left(C_{1}^{n}, T^{n-1}, \epsilon^{n}\right) \\
& +K\left(C_{1}^{n}, T^{n-1}, \epsilon^{n}\right)-K\left(C_{1_{h}}^{n}, T^{n-1}, \epsilon^{n}\right) \\
& +K\left(C_{1_{h}}^{n}, T^{n-1}, \epsilon^{n}\right)-K\left(C_{1_{h}}^{n}, T_{h}^{n-1}, \epsilon^{n}\right) \\
\lesssim & k\|T\|_{W^{1, \infty}}\left\|\epsilon^{n}\right\|_{0, \Omega}+\rho\left\|\nabla\left(C_{1}^{n}-C_{1_{h}}^{n}\right)\right\|_{0, \Omega}\left\|\epsilon^{n}\right\|_{0, \Omega} \\
& +\rho C_{g} M_{C}\left\|\nabla\left(T^{n-1}-T_{h}^{n-1}\right)\right\|_{0, \Omega}\left\|\epsilon^{n}\right\|_{0, \Omega},
\end{aligned}
$$

From where:

$$
\begin{aligned}
& \frac{1}{2 k}\left(\left\|\epsilon^{n}\right\|_{0, \Omega}^{2}-\left\|\epsilon^{n-1}\right\|_{0, \Omega}^{2}+\left\|\epsilon^{n}-\epsilon^{n-1}\right\|_{0, \Omega}^{2}\right)+\lambda\left\|\nabla\left(C_{1}^{n}-C_{1_{h}}^{n}\right)\right\|_{0, \Omega}^{2} \\
& \lesssim\left(h^{\sigma}+k\right)\left\|\epsilon^{n}\right\|_{0, \Omega}+\lambda\left\|\nabla\left(C_{1}^{n}-r_{h} C_{1}^{n}\right)\right\|_{0, \Omega}^{2} \\
&+\left(h^{2 \sigma}+k^{2}\right)+\left(\theta_{8} N M+N M_{C}\right)\left\|\nabla\left(C_{1}^{n}-C_{1_{h}}^{n}\right)\right\|_{0, \Omega}^{2} \\
&+\left(\theta_{9} N M_{C}+\frac{N M_{C}}{2}\right)\left\|\nabla\left(u^{n-1}-u_{h}^{n-1}\right)\right\|_{0, \Omega}^{2} \\
&+k\left\|\epsilon^{n}\right\|_{0, \Omega}+\rho\left\|\nabla\left(C_{1}^{n}-C_{1_{h}}^{n}\right)\right\|_{0, \Omega}\left\|\epsilon^{n}\right\|_{0, \Omega} \\
&+\rho M_{C} C_{g}\left\|\nabla\left(T^{n-1}-T_{h}^{n-1}\right)\right\|_{0, \Omega}\left\|\epsilon^{n}\right\|_{0, \Omega} .
\end{aligned}
$$

From where:

$$
\begin{aligned}
\frac{1}{2 k}\left(\left\|\epsilon^{n}\right\|_{0, \Omega}^{2}-\left\|\epsilon^{n-1}\right\|_{0, \Omega}^{2}+\right. & \left.\left\|\epsilon^{n}-\epsilon^{n-1}\right\|_{0, \Omega}^{2}\right)+\lambda\left\|\nabla\left(C_{1}^{n}-C_{1_{h}}^{n}\right)\right\|_{0, \Omega}^{2} \\
\lesssim & \left(h^{2 \sigma}+k^{2}\right)+\left(\theta_{10}+\theta_{11}+\theta_{12}\right)\left\|\epsilon^{n}\right\|_{0, \Omega}^{2} \\
& +\left(\theta_{8} N M+N M_{C}\right)\left\|\nabla\left(C_{1}^{n}-C_{1_{h}}^{n}\right)\right\|_{0, \Omega}^{2} \\
& +\rho^{2} \frac{M_{C}^{2} C_{g}^{2}}{4 \theta_{12}}\left\|\nabla\left(T^{n-1}-T_{h}^{n-1}\right)\right\|_{0, \Omega}^{2}+\frac{\rho^{2}}{4 \theta_{11}}\left\|\nabla\left(C_{1}^{n}-C_{1_{h}}^{n}\right)\right\|_{0, \Omega}^{2} \\
& \left(\theta_{9} N M_{C}+\frac{N M_{C}}{2}\right)\left\|\nabla\left(u^{n-1}-u_{h}^{n-1}\right)\right\|_{0, \Omega}^{2} .
\end{aligned}
$$

Lemma 5.15. For all constants $\theta_{11}, \theta_{12}, \theta_{16}, \theta_{17}, \theta_{18}, \theta_{19}$, independents of $h$ and $k$, we have:

$$
\left\{\begin{array}{r}
\frac{1}{2 k} \lesssim\left(h^{2 \sigma}+\epsilon^{n}\left\|_{0, \Omega}^{2}-\right\| \epsilon^{n-1}\left\|_{0, \Omega}^{2}+\right\| \epsilon^{n}-\epsilon^{n-1} \|_{0, \Omega}^{2}\right)+\lambda\left\|\nabla\left(C_{2}^{n}-C_{2_{h}}^{n}\right)\right\|_{0, \Omega}^{2} \\
\left(\theta_{16}+\theta_{17}+\theta_{18}+\theta_{19}\right)\left\|\epsilon^{n}\right\|_{0, \Omega}^{2}+\left(\theta_{11} N M+N M_{C}\right)\left\|\nabla\left(C_{2}^{n}-C_{2_{h}}^{n}\right)\right\|_{0, \Omega}^{2} \\
+\rho^{2} \frac{M_{C}^{2} C_{g}^{2}}{\theta_{16}}\left\|\nabla\left(T^{n}-T_{h}^{n-1}\right)\right\|_{0, \Omega}^{2}+\frac{\rho^{2}}{4 \theta_{14}}\left\|\nabla\left(C_{2}^{n}-C_{2_{h}}^{n-1}\right)\right\|_{0, \Omega}^{2} \\
+\frac{\rho^{2}}{4 \theta_{15}}\left\|\nabla\left(C_{1}^{n}-C_{1_{h}}^{n-1}\right)\right\|_{0, \Omega}^{2}+\left(\theta_{12} N M_{C}+\frac{N M_{C}}{2}\right)\left\|\nabla\left(u^{n-1}-u_{h}^{n-1}\right)\right\|_{0, \Omega}^{2} .
\end{array}\right.
$$

Proof. The proof of this lemma is similar to the proof of the previous lemma.

Lemma 5.16. We assume that $N M_{C}<\frac{\lambda}{2}, 6 M_{C}^{2} C_{g}^{2} \leq \lambda$ and $\left(k \leq \frac{1}{4}\right)$. We have:

$$
\left\{\begin{array}{r}
\left\|C_{1}^{n}-C_{1_{h}}^{n}\right\|_{0, \Omega}^{2}+\frac{16}{7} k\left(\lambda-3 \rho^{2}\right)\left\|\nabla\left(C_{1}^{n}-C_{1_{h}}^{n}\right)\right\|_{0, \Omega}^{2} \lesssim k n\left(h^{2 \sigma}+k^{2}\right) \\
+\frac{16 k}{7}\left(\theta_{9} N M_{C}+\frac{N M_{C}}{2}\right) \sum_{i=1}^{n-1}\left\|\nabla\left(u^{i}-u_{h}^{i}\right)\right\|_{0, \Omega}^{2} \\
+\frac{48}{7} \rho^{2} k M_{C}^{2} C_{g}^{2} \sum_{i=1}^{n}\left\|\nabla\left(T^{i}-T_{h}^{i}\right)\right\|_{0, \Omega}^{2} .
\end{array}\right.
$$

Proof. For $\theta_{10}=\theta_{11}=\theta_{12}=\frac{1}{12}, \theta_{8}=\frac{\lambda-2 N M_{C}}{2 N M}$ (because $N M_{C}<\frac{\lambda}{2}$ ), we have:

$$
\begin{aligned}
\frac{1}{2 k}\left(\left\|\epsilon^{n}\right\|_{0, \Omega}^{2}-\left\|\epsilon^{n-1}\right\|_{0, \Omega}^{2}+\left\|\epsilon^{n}-\epsilon^{n-1}\right\|_{0, \Omega}^{2}\right)+\frac{\lambda}{2}\left\|\nabla\left(C_{1}^{n}-C_{1_{h}}^{n}\right)\right\|_{0, \Omega}^{2} & \\
\lesssim & \frac{1}{4}\left\|\epsilon^{n}\right\|_{0, \Omega}^{2}+\left(h^{2 \sigma}+k^{2}\right)+3 \rho^{2} M_{C}^{2} C_{g}^{2}\left\|\nabla\left(T^{n-1}-T_{h}^{n-1}\right)\right\|_{0, \Omega}^{2} \\
& \quad+3 \rho^{2}\left\|\nabla\left(C_{1}^{n}-C_{1_{h}}^{n}\right)\right\|_{0, \Omega}^{2}+\left(\theta_{4} N B+\frac{N B}{2}\right)\left\|\nabla\left(u^{n-1}-u_{h}^{n-1}\right)\right\|_{0, \Omega}^{2} .
\end{aligned}
$$


From where

$$
\begin{aligned}
\frac{1}{2 k}\left(\left\|\epsilon^{n}\right\|_{0, \Omega}^{2}\right)+\frac{\lambda}{2} \sum_{i=1}^{n} & \left\|\nabla\left(C_{1}^{i}-C_{1_{h}}^{i}\right)\right\|_{0, \Omega}^{2} \lesssim \frac{1}{4} \sum_{i=1}^{n}\left\|\epsilon^{i}\right\|_{0, \Omega}^{2}+n\left(h^{2 \sigma}+k^{2}\right) \\
+ & 3 \rho^{2} M_{C}^{2} C_{g}^{2} \sum_{i=1}^{n-1}\left\|\nabla\left(T^{i}-T_{h}^{i}\right)\right\|_{0, \Omega}^{2}+3 \rho^{2} \sum_{i=1}^{n}\left\|\nabla\left(C_{1}^{i}-C_{1_{h}}^{i}\right)\right\|_{0, \Omega}^{2} \\
& +\left(\theta_{9} N M_{C}+\frac{N M_{C}}{2}\right) \sum_{i=1}^{n-1}\left\|\nabla\left(u^{i}-u_{h}^{i}\right)\right\|_{0, \Omega}^{2} .
\end{aligned}
$$

While multiplying by $2 k$ and by using the assumption $k \leq \frac{1}{4}$, we obtain:

$$
\begin{aligned}
\frac{7}{8}\left(\left\|\epsilon^{n}\right\|_{0, \Omega}^{2}\right)+2 k(\lambda & \left.-3 \rho^{2}\right)\left\|\nabla\left(C_{1}^{n}-C_{1_{h}}^{n}\right)\right\|_{0, \Omega}^{2} \lesssim \frac{k}{2} \sum_{i=1}^{n-1}\left\|\epsilon^{i}\right\|_{0, \Omega}^{2} \\
+ & k n\left(h^{2 \sigma}+k^{2}\right)+6 \rho^{2} k M_{C}^{2} C_{g}^{2} \sum_{i=1}^{n}\left\|\nabla\left(T^{i}-T_{h}^{i}\right)\right\|_{0, \Omega}^{2} \\
& +2 k\left(\theta_{9} N M_{C}+\frac{N M_{C}}{2}\right) \sum_{i=1}^{n-1}\left\|\nabla\left(u^{i}-u_{h}^{i}\right)\right\|_{0, \Omega}^{2} .
\end{aligned}
$$

While multiplying by $\frac{8}{7}$, we obtain:

$$
\begin{aligned}
\left(\left\|\epsilon^{n}\right\|_{0, \Omega}^{2}\right)+\frac{16}{7} k(\lambda- & \left.3 \rho^{2}\right)\left\|\nabla\left(C_{1}^{n}-C_{1_{h}}^{n}\right)\right\|_{0, \Omega}^{2} \lesssim \frac{4 k}{7} \sum_{i=1}^{n-1}\left\|\epsilon^{i}\right\|_{0, \Omega}^{2} \\
+ & k n\left(h^{2 \sigma}+k^{2}\right)+\frac{48}{7} \rho^{2} k M_{C}^{2} C_{g}^{2} \sum_{i=1}^{n}\left\|\nabla\left(T^{i}-T_{h}^{i}\right)\right\|_{0, \Omega}^{2} \\
& +\frac{16 k}{7}\left(\theta_{9} N M_{C}+\frac{N M_{C}}{2}\right) \sum_{i=1}^{n-1}\left\|\nabla\left(u^{i}-u_{h}^{i}\right)\right\|_{0, \Omega}^{2} .
\end{aligned}
$$

By using the discret lemma of Gronwal, we obtain:

$$
\begin{aligned}
\left\|\epsilon^{n}\right\|_{0, \Omega}^{2}+\frac{16}{7} k\left(\lambda-3 \rho^{2}\right) & \left\|\nabla\left(C_{1}^{n}-C_{1_{h}}^{n}\right)\right\|_{0, \Omega}^{2} \lesssim\left(k n\left(h^{2 \sigma}+k^{2}\right)\right. \\
+ & \frac{48}{7} \rho^{2} k M_{C}^{2} C_{g}^{2} \sum_{i=1}^{n}\left\|\nabla\left(T^{i}-T_{h}^{i}\right)\right\|_{0, \Omega}^{2} \\
& \left.+\frac{16 k}{7}\left(\theta_{9} N M_{C}+\frac{N M_{C}}{2}\right) \sum_{i=1}^{n-1}\left\|\nabla\left(u^{i}-u_{h}^{i}\right)\right\|_{0, \Omega}^{2}\right) \exp (k n) .
\end{aligned}
$$

The result comes from the triangular inequality:

$$
\begin{array}{r}
\left\|C_{1}^{n}-C_{1_{h}}^{n}\right\|_{0, \Omega}^{2}+\frac{16}{7} k\left(\lambda-3 \rho^{2}\right)\left\|\nabla\left(C_{1}^{n}-C_{1_{h}}^{n}\right)\right\|_{0, \Omega}^{2} \leq\left\|C_{1}^{n}-r_{h} C_{1}^{n}\right\|_{0, \Omega}^{2}+\left\|\epsilon^{n}\right\|_{0, \Omega}^{2} \\
+\frac{16}{7} k\left(\lambda-3 \rho^{2}\right)\left\|\nabla\left(C_{1}^{n}-C_{1_{h}}^{n}\right)\right\|_{0, \Omega}^{2} \\
\lesssim h^{\sigma}\left\|C_{1}\right\|_{L^{\infty}\left(H^{\sigma}\right)}+\left\|\epsilon^{n}\right\|_{0, \Omega}^{2}+k\left\|\nabla\left(C_{1}^{n}-C_{1_{h}}^{n}\right)\right\|_{0, \Omega}^{2} .
\end{array}
$$

Lemma 5.17. We assume that $N M_{C}<\frac{\lambda}{2}, 6 M_{C}^{2} C_{g}^{2} \leq \lambda$ and $\left(k \leq \frac{1}{4}\right)$. We have:

$$
\begin{array}{r}
\quad+\frac{16}{7} k\left(\lambda-3 \rho^{2}\right)\left\|\nabla\left(C_{2}^{n}-C_{2_{h}}^{n}\right)\right\|_{0, \Omega}^{2} \lesssim k n\left(h^{2 \sigma}+k^{2}\right) \\
+\frac{16 k}{7}\left(\theta_{12} N M_{C}+\frac{N M_{C}}{2}\right) \sum_{i=1}^{n-1}\left\|\nabla\left(u^{i}-u_{h}^{i}\right)\right\|_{0, \Omega}^{2} \\
+\frac{256}{7} k M_{C}^{2} C_{g}^{2} \sum_{i=1}^{n}\left\|T^{i}-T_{h}^{i}\right\|_{0, \Omega}^{2}+\frac{64}{7} k \sum_{i=1}^{n}\left\|C_{1}^{i}-C_{1_{h}}^{i}\right\|_{0, \Omega}^{2} .
\end{array}
$$

Proof. For $\theta_{16}=\theta_{17}=\theta_{18}=\theta_{19}=\frac{1}{16}, \theta_{11}=\frac{\lambda-2 N M_{C}}{2 N M}$ (because $N M_{C}<\frac{\lambda}{2}$ ), we have:

$$
\begin{aligned}
\frac{1}{2 k}\left(\left\|\epsilon^{n}\right\|_{0, \Omega}^{2}\right. & \left.-\left\|\epsilon^{n-1}\right\|_{0, \Omega}^{2}+\left\|\epsilon^{n}-\epsilon^{n-1}\right\|_{0, \Omega}^{2}\right)+\frac{\lambda}{2}\left\|\nabla\left(C_{2}^{n}-C_{2_{h}}^{n}\right)\right\|_{0, \Omega}^{2} \lesssim \frac{1}{4}\left\|\epsilon^{n}\right\|_{0, \Omega}^{2} \\
& +\left(h^{2 \sigma}+k^{2}\right)+16 \rho^{2} M_{C}^{2} C_{g}^{2}\left\|\nabla\left(T^{n-1}-T_{h}^{n-1}\right)\right\|_{0, \Omega}^{2}+4 \rho^{2}\left\|\nabla\left(C_{1}^{n}-C_{1_{h}}^{n}\right)\right\|_{0, \Omega}^{2} \\
& +4 \rho^{2}\left\|\nabla\left(C_{2}^{n}-C_{2_{h}}^{n}\right)\right\|_{0, \Omega}^{2}+\left(\theta_{12} N B+\frac{N B}{2}\right)\left\|\nabla\left(u^{n-1}-u_{h}^{n-1}\right)\right\|_{0, \Omega}^{2} .
\end{aligned}
$$

From where

$$
\begin{aligned}
\frac{1}{2 k}\left(\left\|\epsilon^{n}\right\|_{0, \Omega}^{2}\right) & +\frac{\lambda}{2} \sum_{i=1}^{n}\left\|\nabla\left(C_{2}^{i}-C_{2_{h}}^{i}\right)\right\|_{0, \Omega}^{2} \lesssim \frac{1}{4} \sum_{i=1}^{n}\left\|\epsilon^{i}\right\|_{0, \Omega}^{2}+n\left(h^{2 \sigma}+k^{2}\right) \\
& +16 \rho^{2} M_{C}^{2} C_{g}^{2} \sum_{i=1}^{n-1}\left\|\nabla\left(T^{i}-T_{h}^{i}\right)\right\|_{0, \Omega}^{2}+4 \rho^{2} \sum_{i=1}^{n}\left\|\nabla\left(C_{1}^{i}-C_{1_{h}}^{i}\right)\right\|_{0, \Omega}^{2} \\
& +4 \rho^{2} \sum_{i=1}^{n}\left\|\nabla\left(C_{2}^{i}-C_{2_{h}}^{i}\right)\right\|_{0, \Omega}^{2}+\left(\theta_{12} N M_{C}+\frac{N M_{C}}{2}\right) \sum_{i=1}^{n-1}\left\|\nabla\left(u^{i}-u_{h}^{i}\right)\right\|_{0, \Omega}^{2} .
\end{aligned}
$$


While multiplying by $2 k$ and by using the assumption $k \leq \frac{1}{4}$, we obtain:

$$
\begin{aligned}
\frac{7}{8}\left(\left\|\epsilon^{n}\right\|_{0, \Omega}^{2}\right) & +2 k\left(\lambda-4 \rho^{2}\right)\left\|\nabla\left(C_{2}^{n}-C_{2_{h}}^{n}\right)\right\|_{0, \Omega}^{2} \lesssim \frac{k}{2} \sum_{i=1}^{n-1}\left\|\epsilon^{i}\right\|_{0, \Omega}^{2}+k n\left(h^{2 \sigma}+k^{2}\right) \\
& +32 \rho^{2} k M_{C}^{2} C_{g}^{2} \sum_{i=1}^{n}\left\|\nabla\left(T^{i}-T_{h}^{i}\right)\right\|_{0, \Omega}^{2}+8 \rho^{2} k \sum_{i=1}^{n}\left\|\nabla\left(C_{1}^{i}-C_{1_{h}}^{i}\right)\right\|_{0, \Omega}^{2} \\
& +2 k\left(\theta_{12} N M_{C}+\frac{N M_{C}}{2}\right) \sum_{i=1}^{n-1}\left\|\nabla\left(u^{i}-u_{h}^{i}\right)\right\|_{0, \Omega}^{2} .
\end{aligned}
$$

While multiplying by $\frac{8}{7}$, we obtain:

$$
\begin{aligned}
\left(\left\|\epsilon^{n}\right\|_{0, \Omega}^{2}\right) & +\frac{16}{7} k\left(\lambda-4 \rho^{2}\right)\left\|\nabla\left(C_{2}^{n}-C_{2_{h}}^{n}\right)\right\|_{0, \Omega}^{2} \lesssim \frac{4 k}{7} \sum_{i=1}^{n-1}\left\|\epsilon^{i}\right\|_{0, \Omega}^{2}+k n\left(h^{2 \sigma}+k^{2}\right) \\
& +\frac{256}{7} \rho^{2} k M_{C}^{2} C_{g}^{2} \sum_{i=1}^{n}\left\|\nabla\left(T^{i}-T_{h}^{i}\right)\right\|_{0, \Omega}^{2}+\frac{64}{7} \rho^{2} k \sum_{i=1}^{n}\left\|\nabla\left(C_{1}^{i}-C_{1_{h}}^{i}\right)\right\|_{0, \Omega}^{2} \\
& +\frac{16 k}{7}\left(\theta_{12} N M_{C}+\frac{N M_{C}}{2}\right) \sum_{i=1}^{n-1}\left\|\nabla\left(u^{i}-u_{h}^{i}\right)\right\|_{0, \Omega}^{2} .
\end{aligned}
$$

By using the discret lemma of Gronwal, we obtain:

$$
\begin{aligned}
\left\|\epsilon^{n}\right\|_{0, \Omega}^{2}+\frac{16}{7} k\left(\lambda-4 \rho^{2}\right) & \left\|\nabla\left(C_{2}^{n}-C_{2_{h}}^{n}\right)\right\|_{0, \Omega}^{2} \lesssim\left(k n\left(h^{2 \sigma}+k^{2}\right)\right. \\
+ & \frac{256}{7} \rho^{2} k M_{C}^{2} C_{g}^{2} \sum_{i=1}^{n}\left\|\nabla\left(T^{i}-T_{h}^{i}\right)\right\|_{0, \Omega}^{2}+\frac{64}{7} \rho^{2} k \sum_{i=1}^{n}\left\|\nabla\left(C_{1}^{i}-C_{1_{h}}^{i}\right)\right\|_{0, \Omega}^{2} \\
& \left.+\frac{16 k}{7}\left(\theta_{12} N M_{C}+\frac{N M_{C}}{2}\right) \sum_{i=1}^{n-1}\left\|\nabla\left(u^{i}-u_{h}^{i}\right)\right\|_{0, \Omega}^{2}\right) \exp (k n) .
\end{aligned}
$$

The result comes from the triangular inequality:

$$
\begin{array}{r}
\left\|C_{2}^{n}-C_{2_{h}}^{n}\right\|_{0, \Omega}^{2}+\frac{16}{7} k\left(\lambda-4 \rho^{2}\right)\left\|\nabla\left(C_{2}^{n}-C_{2_{h}}^{n}\right)\right\|_{0, \Omega}^{2} \leq\left\|C_{2}^{n}-r_{h} C_{2}^{n}\right\|_{0, \Omega}^{2}+\left\|\epsilon^{n}\right\|_{0, \Omega}^{2} \\
+\frac{16}{7} k\left(\lambda-3 \rho^{2}\right)\left\|\nabla\left(C_{2}^{n}-C_{2_{h}}^{n}\right)\right\|_{0, \Omega}^{2} \\
\lesssim h^{\sigma}\left\|C_{2}\right\|_{L^{\infty}\left(H^{\sigma}\right)}+\left\|\epsilon^{n}\right\|_{0, \Omega}^{2}+k\left\|\nabla\left(C_{2}^{n}-C_{2_{h}}^{n}\right)\right\|_{0, \Omega}^{2} .
\end{array}
$$

Lemma 5.18. For the velocity, we have the following estimate:

$$
\begin{aligned}
& \left\|u^{n}-u_{h}^{n}\right\|_{0, \Omega}^{2}+k\left\|\nabla\left(u^{n}-u_{h}^{n}\right)\right\|_{0, \Omega}^{2} \lesssim\left(k n\left(h^{2 \sigma}+k^{2}\right)+k^{2} n^{2}\left(h^{2 \sigma}+k^{2}\right)\right) \\
& \exp \left(k^{2} n^{2}\left(2\left(\theta_{4} N B+\frac{N B}{2}\right)+\frac{16 k}{7}\left(\theta_{9} N M_{C}+\theta_{12} N M_{C}+N M_{C}\right)\right)\right) .
\end{aligned}
$$

Proof. According to the previous lemmas, we have:

$$
\begin{gathered}
\left\|T^{n}-T_{h}^{n}\right\|_{0, \Omega}^{2}+\lambda k\left\|\nabla\left(T^{n}-T_{h}^{n}\right)\right\|_{0, \Omega}^{2} \lesssim k n\left(h^{2 \sigma}+k^{2}\right)+2 k\left(\theta_{4} N B+\frac{N B}{2}\right) \sum_{i=1}^{n-1}\left\|\nabla\left(u^{i}-u_{h}^{i}\right)\right\|_{0, \Omega}^{2} \\
+8 k \sum_{i=1}^{n}\left\|C_{1}^{i}-C_{1_{h}}^{i}\right\|_{0, \Omega}^{2}+8 k \sum_{i=1}^{n}\left\|C_{2}^{i}-C_{2_{h}}^{i}\right\|_{0, \Omega}^{2}, \\
\left\|C_{1}^{n}-C_{1_{h}}^{n}\right\|_{0, \Omega}^{2}+\frac{16}{7} k\left(\lambda-3 \rho^{2}\right)\left\|\nabla\left(C_{1}^{n}-C_{1_{h}}^{n}\right)\right\|_{0, \Omega}^{2} \lesssim k n\left(h^{2 \sigma}+k^{2}\right) \\
+\frac{16 k}{7}\left(\theta_{9} N M_{C}+\frac{N M_{C}}{2}\right) \sum_{i=1}^{n-1}\left\|\nabla\left(u^{i}-u_{h}^{i}\right)\right\|_{0, \Omega}^{2} \\
+\frac{48}{7} k M_{C}^{2} C_{g}^{2} \sum_{i=1}^{n}\left\|T^{i}-T_{h}^{i}\right\|_{0, \Omega}^{2} .
\end{gathered}
$$

and

$$
\begin{array}{r}
\quad+\frac{16}{7} k\left(\lambda-3 \rho^{2}\right)\left\|\nabla\left(C_{2}^{n}-C_{2_{h}}^{n}\right)\right\|_{0, \Omega}^{2} \lesssim k n\left(h^{2 \sigma}+k^{2}\right) \\
+\frac{16 k}{7}\left(\theta_{12} N M_{C}+\frac{N M_{C}}{2}\right) \sum_{i=1}^{n-1}\left\|\nabla\left(u^{i}-u_{h}^{i}\right)\right\|_{0, \Omega}^{2} \\
+\frac{256}{7} k M_{C}^{2} C_{g}^{2} \sum_{i=1}^{n}\left\|T^{i}-T_{h}^{i}\right\|_{0, \Omega}^{2}+\frac{64}{7} k \sum_{i=1}^{n}\left\|C_{1}^{i}-C_{1_{h}}^{i}\right\|_{0, \Omega}^{2} .
\end{array}
$$


By summing, we obtain:

$$
\begin{aligned}
\| T^{n} & -T_{h}^{n}\left\|_{0, \Omega}^{2}+\right\| C_{1}^{n}-C_{1_{h}}^{n}\left\|_{0, \Omega}^{2}+\right\| C_{2}^{n}-C_{2_{h}}^{n}\left\|_{0, \Omega}^{2}+\lambda k\right\| \nabla\left(T^{n}-T_{h}^{n}\right) \|_{0, \Omega}^{2} \\
& +\frac{16}{7} k\left(\lambda-3 \rho^{2}\right)\left\|\nabla\left(C_{1}^{n}-C_{1_{h}}^{n}\right)\right\|_{0, \Omega}^{2}+\frac{16}{7} k\left(\lambda-3 \rho^{2}\right)\left\|\nabla\left(C_{2}^{n}-C_{2_{h}}^{n}\right)\right\|_{0, \Omega}^{2} \lesssim k n\left(h^{2 \sigma}+k^{2}\right) \\
& +\left(2 k\left(\theta_{4} N B+\frac{N B}{2}\right)+\frac{16 k}{7}\left(\theta_{9} N M_{C}+\theta_{12} N M_{C}+N M_{C}\right)\right) \\
& \times \sum_{i=1}^{n-1}\left\|\nabla\left(u^{i}-u_{h}^{i}\right)\right\|_{0, \Omega}^{2}+\frac{120}{7} k \sum_{i=1}^{n}\left\|C_{1}^{i}-C_{1_{h}}^{i}\right\|_{0, \Omega}^{2} \\
& +8 k \sum_{i=1}^{n}\left\|C_{2}^{i}-C_{2_{h}}^{i}\right\|_{0, \Omega}^{2}+\frac{304}{7} k M_{C}^{2} C_{g}^{2} \sum_{i=1}^{n}\left\|T^{i}-T_{h}^{i}\right\|_{0, \Omega}^{2} .
\end{aligned}
$$

If we take $w=\max \left(\frac{120}{7} k, \frac{304}{7} k M_{C}^{2} C_{g}^{2}\right)$, we obtain

$$
\begin{aligned}
& \left\|T^{n}-T_{h}^{n}\right\|_{0, \Omega}^{2}+\left\|C_{1}^{n}-C_{1_{h}}^{n}\right\|_{0, \Omega}^{2}+\left\|C_{2}^{n}-C_{2_{h}}^{n}\right\|_{0, \Omega}^{2}+\frac{\lambda k}{1-w}\left\|\nabla\left(T^{n}-T_{h}^{n}\right)\right\|_{0, \Omega}^{2}+\frac{16}{7(1-w)} k\left(\lambda-3 \rho^{2}\right) \\
& \quad \times\left\|\nabla\left(C_{1}^{n}-C_{1_{h}}^{n}\right)\right\|_{0, \Omega}^{2}+\frac{16}{7(1-w)} k\left(\lambda-3 \rho^{2}\right)\left\|\nabla\left(C_{2}^{n}-C_{2_{h}}^{n}\right)\right\|_{0, \Omega}^{2} \lesssim \frac{1}{1-w} k n\left(h^{2 \sigma}+k^{2}\right) \\
& \quad+\frac{1}{1-w}\left(2 k\left(\theta_{4} N B+\frac{N B}{2}\right)+\frac{16 k}{7}\left(\theta_{9} N M_{C}+\theta_{12} N M_{C}+N M_{C}\right)\right) \sum_{i=1}^{n-1}\left\|\nabla\left(u^{i}-u_{h}^{i}\right)\right\|_{0, \Omega}^{2} \\
& \quad+\frac{w}{1-w}\left(\sum_{i=1}^{n}\left(\left\|C_{1}^{i}-C_{1_{h}}^{i}\right\|_{0, \Omega}^{2}+\left\|C_{2}^{i}-C_{2_{h}}^{i}\right\|_{0, \Omega}^{2}+\left\|T^{i}-T_{h}^{i}\right\|_{0, \Omega}^{2}\right)\right) .
\end{aligned}
$$

By using the Gronwal lemma with

$$
\begin{gathered}
a_{n}=\left\|T^{n}-T_{h}^{n}\right\|_{0, \Omega}^{2}+\left\|C_{1}^{n}-C_{1_{h}}^{n}\right\|_{0, \Omega}^{2}+\left\|C_{2}^{n}-C_{2_{h}}^{n}\right\|_{0, \Omega}^{2}, \\
b_{n}=\frac{\lambda k}{1-w}\left\|\nabla\left(T^{n}-T_{h}^{n}\right)\right\|_{0, \Omega}^{2}+\frac{16}{7(1-w)} k\left(\lambda-3 \rho^{2}\right)\left\|\nabla\left(C_{1}^{n}-C_{1_{h}}^{n}\right)\right\|_{0, \Omega}^{2}+\frac{16}{7(1-w)} k\left(\lambda-3 \rho^{2}\right) \\
\times\left\|\nabla\left(C_{2}^{n}-C_{2_{h}}^{n}\right)\right\|_{0, \Omega}^{2}
\end{gathered}
$$

and

$$
\begin{aligned}
c_{n}= & \frac{1}{1-w} k n\left(h^{2 \sigma}+k^{2}\right)+\frac{1}{1-w}\left(2 k\left(\theta_{4} N B+\frac{N B}{2}\right)+\frac{16 k}{7}\left(\theta_{9} N M_{C}+\theta_{12} N M_{C}+N M_{C}\right)\right) \\
& \times \sum_{i=1}^{n-1}\left\|\nabla\left(u^{i}-u_{h}^{i}\right)\right\|_{0, \Omega}^{2},
\end{aligned}
$$

we obtain:

$$
\begin{aligned}
& \left\|T^{n}-T_{h}^{n}\right\|_{0, \Omega}^{2}\left\|C_{1}^{n}-C_{1_{h}}^{n}\right\|_{0, \Omega}^{2}+\left\|C_{2}^{n}-C_{2_{h}}^{n}\right\|_{0, \Omega}^{2}+\frac{\lambda k}{1-w}\left\|\nabla\left(T^{n}-T_{h}^{n}\right)\right\|_{0, \Omega}^{2}+\frac{16}{7(1-w)} k\left(\lambda-3 \rho^{2}\right) \\
& \quad \times\left\|\nabla\left(C_{1}^{n}-C_{1_{h}}^{n}\right)\right\|_{0, \Omega}^{2}+\frac{16}{7(1-w)} k\left(\lambda-3 \rho^{2}\right)\left\|\nabla\left(C_{2}^{n}-C_{2_{h}}^{n}\right)\right\|_{0, \Omega}^{2} \lesssim\left(\frac{1}{1-w} k n\left(h^{2 \sigma}+k^{2}\right)\right. \\
& \left.\quad+\frac{1}{1-w}\left(2 k\left(\theta_{4} N B+\frac{N B}{2}\right)+\frac{16 k}{7}\left(\theta_{9} N M_{C}+\theta_{12} N M_{C}+N M_{C}\right)\right) \sum_{i=1}^{n-1}\left\|\nabla\left(u^{i}-u_{h}^{i}\right)\right\|_{0, \Omega}^{2}\right) \\
& \quad \times \exp \left(\frac{w n}{1-w}\right) .
\end{aligned}
$$

We have:

$$
\begin{aligned}
\| u^{n}- & u_{h}^{n}\left\|_{0, \Omega}^{2}+k\right\| \nabla\left(u^{n}-u_{h}^{n}\right) \|_{0, \Omega}^{2} \lesssim k n\left(h^{2 \sigma}+k^{2}\right)+k\left(k n \frac{1}{1-w}\left(h^{2 \sigma}+k^{2}\right)\right. \\
& \left.\quad+\frac{1}{1-w}\left(2 k\left(\theta_{4} N B+\frac{N B}{2}\right)+\frac{16 k}{7}\left(\theta_{9} N M_{C}+\theta_{12} N M_{C}+N M_{C}\right)\right)\right) \sum_{j=2}^{n} \sum_{i=1}^{j-1}\left\|\nabla\left(u^{i}-u_{h}^{i}\right)\right\|_{0, \Omega}^{2} .
\end{aligned}
$$


Finally, we have:

$$
\begin{aligned}
& \left\|u^{n}-u_{h}^{n}\right\|_{0, \Omega}^{2}+k\left\|\nabla\left(u^{n}-u_{h}^{n}\right)\right\|_{0, \Omega}^{2} \lesssim\left(k n\left(h^{2 \sigma}+k^{2}\right)+k^{2} n^{2}\left(h^{2 \sigma}+k^{2}\right)\right) \\
& \exp \left(k^{2} n^{2}\left(2\left(\theta_{4} N B+\frac{N B}{2}\right)+\frac{16 k}{7}\left(\theta_{9} N M_{C}+\theta_{12} N M_{C}+N M_{C}\right)\right)\right) .
\end{aligned}
$$

Lemma 5.19. We have the following at the same time for the temperature and for the concentrations :

$$
\left\|T^{n}-T_{h}^{n}\right\|_{0, \Omega}^{2}+\frac{\lambda k}{1-w}\left\|\nabla\left(T^{n}-T_{h}^{n}\right)\right\|_{0, \Omega}^{2} \lesssim\left(h^{2 \sigma}+k^{2}\right)\left(k n+k^{2} n^{2}+k^{3} n^{3}\right)
$$

and

$$
\left\|C_{i}^{n}-C_{i_{h}}^{n}\right\|_{0, \Omega}^{2}+\frac{16}{7(1-w)} k\left(\lambda-3 \rho^{2}\right)\left\|\nabla\left(C_{i}^{n}-C_{i_{h}}^{n}\right)\right\|_{0, \Omega}^{2} \lesssim\left(h^{2 \sigma}+k^{2}\right)\left(k n+k^{2} n^{2}+k^{3} n^{3}\right) . \quad(i=1,2)
$$

Proof. We have

$$
\begin{aligned}
& \left\|T^{n}-T_{h}^{n}\right\|_{0, \Omega}^{2}+\left\|C_{1}^{n}-C_{1_{h}}^{n}\right\|_{0, \Omega}^{2}+\left\|C_{2}^{n}-C_{2_{h}}^{n}\right\|_{0, \Omega}^{2}+\frac{\lambda k}{1-w}\left\|\nabla\left(T^{n}-T_{h}^{n}\right)\right\|_{0, \Omega}^{2}+\frac{16}{7(1-w)} k\left(\lambda-3 \rho^{2}\right) \\
& \quad \times\left\|\nabla\left(C_{1}^{n}-C_{1_{h}}^{n}\right)\right\|_{0, \Omega}^{2}+\frac{16}{7(1-w)} k\left(\lambda-3 \rho^{2}\right)\left\|\nabla\left(C_{2}^{n}-C_{2_{h}}^{n}\right)\right\|_{0, \Omega}^{2} \lesssim \frac{1}{1-w} k n\left(h^{2 \sigma}+k^{2}\right) \\
& \quad+\frac{1}{1-w}\left(2 k\left(\theta_{4} N B+\frac{N B}{2}\right)+\frac{16 k}{7}\left(\theta_{9} N M_{C}+\theta_{12} N M_{C}+N M_{C}\right)\right) \sum_{i=1}^{n-1}\left\|\nabla\left(u^{i}-u_{h}^{i}\right)\right\|_{0, \Omega}^{2} .
\end{aligned}
$$

According to the previous lemma, we can remark that:

$$
k \sum_{i=1}^{n-1}\left\|\nabla\left(u^{i}-u_{h}^{i}\right)\right\|_{0, \Omega}^{2} \lesssim k n\left(k n\left(h^{2 \sigma}+k^{2}\right)+k^{2} n^{2}\left(h^{2 \sigma}+k^{2}\right)\right)
$$

Therefore:

$$
\begin{aligned}
\left\|T^{n}-T_{h}^{n}\right\|_{0, \Omega}^{2} & +\left\|C_{1}^{n}-C_{1_{h}}^{n}\right\|_{0, \Omega}^{2}+\left\|C_{2}^{n}-C_{2_{h}}^{n}\right\|_{0, \Omega}^{2}+\frac{\lambda k}{1-w}\left\|\nabla\left(T^{n}-T_{h}^{n}\right)\right\|_{0, \Omega}^{2}+\frac{16}{7(1-w)} k\left(\lambda-3 \rho^{2}\right) \\
& \times\left\|\nabla\left(C_{1}^{n}-C_{1_{h}}^{n}\right)\right\|_{0, \Omega}^{2}+\frac{16}{7(1-w)} k\left(\lambda-3 \rho^{2}\right)\left\|\nabla\left(C_{2}^{n}-C_{2_{h}}^{n}\right)\right\|_{0, \Omega}^{2} \lesssim \frac{1}{1-w} n\left(h^{2 \sigma}+k^{2}\right) \\
& +\frac{1}{1-w}\left(2 k\left(\theta_{4} N B+\frac{N B}{2}\right)+\frac{16 k}{7}\left(\theta_{9} N M_{C}+\theta_{12} N M_{C}+N M_{C}\right)\right) \\
& n\left(k n\left(h^{2 \sigma}+k^{2}\right)+k^{2} n^{2}\left(h^{2 \sigma}+k^{2}\right)\right)
\end{aligned}
$$

Conclusion:

$$
\left\|T^{n}-T_{h}^{n}\right\|_{0, \Omega}^{2}+\frac{\lambda k}{1-w}\left\|\nabla\left(T^{n}-T_{h}^{n}\right)\right\|_{0, \Omega}^{2} \lesssim\left(h^{2 \sigma}+k^{2}\right)\left(k n+k^{2} n^{2}+k^{3} n^{3}\right)
$$

We have also:

$$
\left\|C_{i}^{n}-C_{i_{h}}^{n}\right\|_{0, \Omega}^{2}+\frac{16}{7(1-w)} k\left(\lambda-3 \rho^{2}\right)\left\|\nabla\left(C_{i}^{n}-C_{i_{h}}^{n}\right)\right\|_{0, \Omega}^{2} \lesssim\left(h^{2 \sigma}+k^{2}\right)\left(k n+k^{2} n^{2}+k^{3} n^{3}\right) \quad(i=1,2)
$$

Finally, from the previous lemmas, we are able to state our following mean result:

Theorem 5.2. Assume that:

$$
N A<\nu+N M, \quad N B<\frac{\lambda}{2}, \quad N M_{C}<\frac{\lambda}{2}
$$

and

$$
16 M_{C}^{2} C_{g}^{2}<\min \left(\lambda, \frac{\lambda}{2 \rho^{2}}\right), \quad k \leq \frac{1}{4}, \quad w=\max \left(\frac{120}{7} k, \frac{304}{7} k M_{C}^{2} C_{g}^{2}\right)<1 .
$$

If the solution of the problem $(P)$ admits the following regularity:

$$
\left\{\begin{array}{l}
u \in W^{2, \infty}\left(0, t,\left(L^{2}(\Omega)\right)^{d}\right) \cap W^{1, \infty}\left(0, t,\left(H^{1}(\Omega)\right)^{d}\right) \cap L^{\infty}\left(0, t,\left(H^{1+\sigma}(\Omega)\right)^{d}\right), \\
p \in L^{\infty}\left(0, t, H^{\sigma}(\Omega)\right), \\
T, C_{1}, C_{2} \in W^{2, \infty}\left(0, t,\left(L^{2}(\Omega)\right)\right) \cap W^{1, \infty}\left(0, t, H^{1}(\Omega)\right) \cap L^{\infty}\left(0, t, H^{1+\sigma}(\Omega)\right),
\end{array}\right.
$$


then we will have the following error estimates:

$$
\left\|u^{n}-u_{h}^{n}\right\|_{0, \Omega}^{2}+k\left\|\nabla\left(u^{n}-u_{h}^{n}\right)\right\|_{0, \Omega}^{2} \lesssim\left(h^{2 \sigma}+k^{2}\right)\left(k n+k^{2} n^{2}\right)
$$

for the velocity,

$$
\left\|p^{n}-p_{h}^{n}\right\|_{0, \Omega}^{2} \lesssim\left(h^{2 \sigma}+k^{2}\right)\left(k n+k^{2} n^{2}+k^{3} n^{3}\right)
$$

for the pressure,

$$
\left\|T^{n}-T_{h}^{n}\right\|_{0, \Omega}^{2}+\frac{\lambda k}{1-w}\left\|\nabla\left(T^{n}-T_{h}^{n}\right)\right\|_{0, \Omega}^{2} \lesssim\left(h^{2 \sigma}+k^{2}\right)\left(k n+k^{2} n^{2}+k^{3} n^{3}\right)
$$

for the temperature and

$$
\left\|C_{i}^{n}-C_{i_{h}}^{n}\right\|_{0, \Omega}^{2}+\frac{16}{7(1-w)} k\left(\lambda-3 \rho^{2}\right)\left\|\nabla\left(C_{i}^{n}-C_{i_{h}}^{n}\right)\right\|_{0, \Omega}^{2} \lesssim\left(h^{2 \sigma}+k^{2}\right)\left(k n+k^{2} n^{2}+k^{3} n^{3}\right), \quad(i=1,2)
$$

for the concentrations.

\section{REFERENCES}

[1] A. Agouzal, K. Allali, Numerical analysis of reaction front propagation model under Boussinesq approximation, Math. Methods Appl. Sci., 26:18 (2003), 1529-1572.

[2] B. Achchab, A. Agouzal, K. Allali, Finite elements approximation of two-step reaction combustion model, Int. J. Pure Appl. Math., 63:4 (2010), 379-407.

[3] K. Allali, J. Pojman, V. Volpert, Influence of Vibrations on Convective Instability of Polymerization Fronts, Journal of Engineering Mathematics, 41:1 (2001), 13-31.

[4] Y. Bai, T.R. Sosnick, L. Mayne, S.W. Englander, Protein folding intermediates: native-state hydrogen exchange, Science, 269:5221 (1995), 192-197.

[5] S.W. Benson, The Foundations of Chemical Kinetics, McGraw-Hill, New York, 1960.

[6] M. Boudart, Kinetics of Chemical Processes, Prentice-Hall, Englewood Cliffs, NJ, 1968.

[7] F. Brezzi, M. Fortin, Mixed and Hybrid Finite Element Methods, Springer Verlag, New York, 1991.

[8] P.G. Ciarlet, The Finite Element Method for Elliptic Problems, North-Holland, Amsterdam, New York, Oxford, 1978.

[9] W.A. Eaton, V. Muoz, S.J. Hagen, G.S. Jas, L.J. Lapidus, E.R. Henry, J. Hofrichter, Fast Kinetics and Mechanisms in Protein Folding, Annual Review of Biophysics and Biomolecular, 29 (2000), 327-359.

[10] V. Girault, P.A. Raviart, Finite Element Approximation of the Navier-Stokes Equations, Springer-Verlag, Berlin, Heidelberg, New-York, 1986.

[11] J. Guo, A.C. Lua, Kinetic study on pyrolytic process of oil-palm solid waste using two-step consecutive reaction model, Biomass and Bioenergy, 20 (2001), 223-233.

[12] O. Maneley, M. Marion, R. Temam, Equation of Combustion in The Presence of Complex Chemistry, Indiana University Mathematics Journal, 42 1993, 941-967.

[13] S.B. Margolis, B.J. Matkowsky, Flame Propagation with a Sequential Reaction Mechanism, SIAM Journal on Applied Mathematics, 42:6 (1982), 1175-1188.

[14] B.J. Matkowsky, G.I. Sivashinsky, An asymptotic derivation of two models in flame theory associated with the constant density approximation, SIAM J. Appl. Math., 37 (1997), 686-699.

[15] P.A. Ramachandran, R. Krishna, C.B. Panchal, Analysis of immobilised multi-enzyme reaction systems with michaelis-menten kinetics, Journal of Applied Chemistry and Biotechnology, 26:4 (1976), 214-224.

[16] M. Tsuji, M. Kawahara, K. Noda, M. Senda, H. Sako, N. Kamo, T. Kawahara, K.S. Kamarudin, Photochemical removal of $\mathrm{NO}(2)$ by using 172-nm Xe(2) excimer lamp in $\mathrm{N}(2)$ or air at atmospheric pressure, J. Hazard Mater, 162:2-3 (2009), 1025-33.

[17] V. Volpert, S.V. Petrovskii, Reaction-diffusion waves in biology, Physics of Life Reviews, 6:4(2009), $267-310$.

[18] A. Volpert, Vit. Volpert, Vl. Volpert, Travelling wave solutions of parabolic systems, AMS, Providence, 1994.

[19] Ya.B. Zeldovich, G.I. Barenblatt, G.I. Librovich, G.M. Makhviladze, The mathematical theory of combustion and explosions, Plenum, New York, 1985. 